\title{
Lapurdum
}

Euskal ikerketen aldizkaria | Revue d'études basques |

Revista de estudios vascos | Basque studies review

$3 \mid 1998$

Numéro III

\section{Sur quelques similitudes toponymiques galaïco- basques et le problème que posent certaines d'entre elles}

\section{Hector Iglesias}

\section{(2) OpenEdition \\ 12 Journals}

\section{Édition électronique}

URL : https://journals.openedition.org/lapurdum/1662

DOI : 10.4000/lapurdum. 1662

ISSN : 1965-0655

Éditeur

IKER

\section{Édition imprimée}

Date de publication : 1 octobre 1998

Pagination : 1-27

ISBN : 2-84127-152-8

ISSN : $1273-3830$

\section{Référence électronique}

Hector Iglesias, «Sur quelques similitudes toponymiques galaïco-basques et le problème que posent certaines d'entre elles », Lapurdum [En ligne], 3 | 1998, mis en ligne le 01 septembre 2010, consulté le 24 février 2022. URL : http://journals.openedition.org/lapurdum/1662 ; DOI : https://doi.org/10.4000/ lapurdum. 1662 


\title{
Hector IGLESIAS
}

\section{SUR QUELQUES SIMILITUDES TOPONYMIQUES GALAÏCO-BASQUES ET LE PROBLËME QUE POSENT CERTAINES D'ENTRE ELLES}

\begin{abstract}
Bien que les études toponymiques concernant la Galice aient toujours été beaucoup moins nombreuses que celles ayant pour objet le Pays Basque ou la Catalogne, il existe néanmoins certains articles et ouvrages linguistiques de grande qualité. Ces derniers mentionnent plusieurs noms de lieux galiciens, particulièrement ceux d'origine latino-romane ainsi que ceux issus du germanique, les principaux travaux étant surtout ceux de Joseph M. Piel. Les travaux de Joan Coromines, dont l'œuvre est pourtant considérable, n'ont abordé que quelques rares points de toponymie galicienne'.
\end{abstract}

On peut dire que les études toponymiques se trouvent actuellement en Galice dans un état moins avancé que dans d'autres régions d'Europe, vraisemblablement pour des raisons que nous aborderons plus loin. Dans le domaine de la toponymie dite pré-indo-européenne, il faut mentionner un court, mais fort intéressant, article de J. Gifford. L'auteur y souligne, sans pour autant les commenter, plusieurs similitudes présumées entre certains toponymes navarrais et certains toponymes galiciens.

Au début du siècle, D. Ramón Menéndez $\mathrm{Pidal}^{2}$, le maître de la philologie espagnole, avait déjà signalé que certains suffixes tels que -barre, -toi et -oi apparaissaient dans divers endroits de la péninsule, tantôt en Galice et dans les Asturies, tantôt dans les Pyrénées occidentales. En outre, plusieurs auteurs, dont Henri Gavel ${ }^{3}$ et Fredrick Jungemann, entre autres, avaient également noté certains parallélismes phonétiques entre ces régions pyrénéennes et cantabriques. Ces connexités phonétiques et toponymiques cantabro-pyrénéennes, et en particuliers les basco-galaïques, posent certains problèmes, relativement complexes, qui n'ont toujours pas été résolus.

En ce qui concerne la Galice pré-romaine, plusieurs faits sont connus. On sait que les Galiciens sont mentionnés pour la première fois au cours du $\mathrm{II}^{e}$ siècle av. J.-C., manifestement vers 137 av. J.-C. Ils sont appelés par les auteurs gréco-latins Callaici, le nom ayant été rapproché, entre autres, de celui des Galates. Mais Julio Caro Baroja $a^{5}$, citant Émile Hübner, doutait de la relation. On croit savoir, toujours d'après les travaux de Julio Caro Baroja, que chez les Artabres ou Artabri, un peuple galaïque qui habitait l'actuelle province de La Corogne, la ville du même nom étant alors vraisemblablement appelée Portus magnus artabrorum, les femmes partaient à la guerre pendant que les hommes restaient à la maison ${ }^{6}$. En outre, grâce à la découverte de plusieurs inscriptions apparaissant sur des stèles 
funéraires, on connaît le nom de plusieurs divinités galaïques pré-latines ${ }^{7}$ : Endovelico, Baraeco, Bandue, Cusuneneoeco, Iupiter Andero, etc.

D'un point de vue linguistique, on sait également que le fleuve appelé Le Miño - le plus important de Galice, en galicien O Miño - était couramment appelé par les Galiciens de l'Antiquité Bainis ou Baenis (Strabon, Géographie, III, 3, 4) ou Baites (Appien, Iberica, 73), le nom devant être comparé à l'ancien nom du Guadalquivir, c'est-à-dire autrefois Baitis, nom qui continue parfois à être utilisé en Espagne sous la forme Betis. Dans ces noms l'élément bai-, ayant vraisemblablement le sens hydronymique de "cours d'eau", est peut-être identique à celui qu'on trouve dans le nom Baigorry, uaigorri, 1072 et probablement aussi dans celui de Bayonne, baiona, 1063 ainsi que dans le vocable basque ibai, "rivière, fleuve". La voyelle initiale $i$ - serait, d'après Hugo Schuchardt $t^{8}$, un ancien préfixe devenu plus tard partie intégrante du thème, sa valeur originelle n'étant plus reconnaissable, d'où une évolution bai > ibai.

On sait qu'il y avait parmi la nation des Galaïques plusieurs tribus ou peuples qui n'étaient pas d'origine celtique. Les dires de Pline l'Ancien (Histoire Naturelle, Livre III, 13) qui, d'après Julio Caro Baroja, faisait toujours preuve d'une grande curiosité et savait vraisemblablement distinguer les langues celtiques de celles qui ne l'étaient pas, l'indiquent : Lucensis conuentus populorum est sedecim, praeter Celticos et Lemauos ignobilium ac barbarae appellationis, sed liberorum capitum ferme CLXVIm, c'est-à-dire "I'assemblée [territoriale] luquoise [représentant les Galaïques luquois, c'est-à-dire à l'époque grosso modo le territoire galicien actuel], outre les Celtes et les Lemaves, comprend seize peuples inconnus ayant des noms barbares, mais constituant néanmoins une population de 166.000 individus libres".

Cela, entre autres, amena Luis Michelena à penser qu'il faudrait "desceltizar tal vez hasta cierto punto Galicia" 9 et celtiser davantage l'Aragon. Par ailleurs, Julio Caro Baroja ${ }^{10}$ ne voyait pas dans les dires de Strabon un simple caprice de cet auteur grec. En effet, ce dernier (Géographie, Livre III, 3, 7), après avoir détaillé les us et coutumes des Lusitaniens de l'Antiquité, ajoute à un moment donné : "Tel est le genre de vie, comme je l'ai dit, des populations montagnardes, j'entends par là celles qui jalonnent le côté nord de l'Tbérie, à savoir les Callaïques, les Astures et les Cantabres, jusqu'au pays des Vascones et au Mont Pyréné. Tous, en effet, vivent de la mëme manière".

Les problèmes, qui ne font pas l'objet de notre article, que pose l'existence de ces peuples du nord-ouest de la péninsule ibérique sont nombreux et relativement ardus.

J. Gifford se contente la plupart du temps de signaler avant tout des ressemblances dans le domaine de la toponymie pré-indo-européenne. En essayant d'élargir le champ de recherche, en y incluant les noms latino-romans et ceux d'origine germanique, nous présentons une liste non-exhaustive de toponymes qui se répètent ou semblent se répéter en Galice et en Pays Basque, parfois en Béarn ou en Aragon, voire présentent quelques affinités. Chaque fois qu'une similitude présumée a été notée par J. Gifford nous l'indiquons expressément. Les autres connexités présumées sont le fruit de nos recherches.

Enfin, quelques précisions s'imposent : la Galice compte actuellement, comme le rappelle Ana Isabel Boullón, environ 60000 documents médiévaux. Ce 
patrimoine documentaire est considéré comme étant l'un des plus riches de la péninsule ibérique. Cependant, un grand nombre de cartulaires, représentant des milliers d'archives, n'a toujours pas été publié. C'est le cas des recueils de chartes de Caaveiro, Celanova, Lugo, Oia, Ribadavia, etc. Cela a pour conséquence, entre autres, de ne pas connaitre la ou les formes anciennes d'un grand nombre de toponymes, ces derniers ayant la plupart du temps une allure très archaique et étant souvent difficiles à expliquer. Leur interprétation est même parfois impossible.

En ce qui concerne le pays à proprement parler, les villes sont peu nombreuses et en général peu peuplées, excepté La Corogne qui compte plus de 200000 habitants. La Galice est séparée de l'Espagne par une série ininterrompue de chaines de montagnes dont l'altitude oscille entre $1500 \mathrm{~m}$. et $2000 \mathrm{~m}$., formant ainsi une sorte de barrière naturelle qui explique peut-être, en partie du moins, l'isolement dans lequel a vécu ce pays jusqu'à une date relativement récente.

Le patrimoine toponymique galicien est étonnamment riche : en ce qui concerne uniquement la toponymie majeure (noms de pays, de vallées, de communes et de hameaux), les plus de 30000 noms de lieux figurant dans la nomenclature officielle publiée par le Gouvernement de Galice représentent presque la moitié de la toponymie majeure de l'État espagnol ${ }^{13}$. En outre, le statut d'autonomie dont jouit ce pays stipule que seules les formes galiciennes des toponymes ont une existence officielle ${ }^{12}$.

La langue galicienne est officielle dans le pays et sa connaissance est obligatoire, le statut d'autonomie précisant que savoir le galicien est un droit mais également un devoir. Cette langue présente généralement un caractère archaïque, sa phonétique étant considérée très conservatrice. C'est pourquoi, avant toute étude toponymique, il est utile de citer, ne serait-ce que de façon sommaire, quelques faits de phonétique galicienne parmi les plus typiques et leurs affinités avec, entre autres, le gascon et le basque :

1) en galicien (comme en basque et en gascon, entre autres) $v$ passe invariablement à $b$ quelle que soit sa position ;

2) à l'intérieur d'un mot $-f$ - passe à $-b$ - : defensa $>$ debesa, trifoliu $>$ trebo, etc., ce qui arrive aussi en basque où les changements $-f_{-}>-f-$ et $-f->-p$ - semblent être moins anciens que ceux $-f_{-}>-b-$;

3 ) un des changements les plus caractéristiques est la chute quasi automatique en galicien de tout $l$ intervocalique : insula $>$ insua, etc.; mais parfois, comme en basque, il passe à $-r$ - : cumulu > cómaro, etc. (en gascon - $l$ - se maintient en général); au surplus, les $-r$ - étymologiques ont tendance dans le langage quotidien à disparaître entre voyelles : queres > ques, "tu veux", eres > es, "tu es", etc., ce qui est aussi le cas dans le basque parlé et systématique en souletin où les -rintervocaliques, étymologiques ou non, ont disparu ;

4) en galicien lat. - $l l$ - passe à $-l-$, ce qui est le cas du basque ; en revanche, en gascon il passe à $-r$ - et en castillan il se palatalise ;

5) en galicien tous les $-n$ - intervocaliques disparaissent comme en portugais, en basque et en gascon, excepté dans le suffixe lat. -inu qui passe en galicien à -iño et dans le groupe $n y$, avec semi-voyelle, qui passe à $\tilde{n}$, comme en basque. C'est là une des principales caractéristiques de ces quatre langues, cela étant peutêtre dû à un substrat commun, mais ce n'est pas sûr (cf. Rohlfs); 
6) en galicien, en basque et en gascon lat. -nn-passe à - $n$-; en castillan, il passe à $-\tilde{n}_{-}^{-}$;

7) en galicien, en basque et en castillan le groupe -nd-se maintient; il passe à $-n$ - en gascon et en catalan (et probablement aussi en aragonais, mais ce n'est pas l'avis de G. Rohlfs);

8) En gascon, en castillan, en catalan, en basque et parfois en galicien, le groupe $-m b$ - se réduit à $-m$ - ;

9) en galicien, comme en gascon, la consonne $r$ présente une instabilité importante, ce qui est à l'origine de nombreuses métathèses dans ces deux langues;

10) en galicien, comme en basque, l'épenthèse d'un $i$ consonne intervocalique, pour détruire des hiratus, est très fréquente ;

11) en galicien, la consonne $r$ à l'initiale est parfois précédée de la voyelle prothétique $a$, c'est-à-dire qu'on a $r$ - > arr-, d'où gal. recuar et arrecuar, "reculer"; rodeo et arrodeo, "détour", etc. ; en asturien cette voyelle prothétique est encore plus. fréquente : arrodiu, arrodéu, arrodión, "détour", arrezar et rezar, "prier" ; arrezu el rezu, "prière", c c'est également le cas du gascon (et parfois, mais très rarement, de l'ancien aragonais) où son apparition est constante jusqu'au Bassin d'Arcachon (cf. G. Rohlfs) et c'est également, et avant tout, une des principales caractéristiques phonétiques de la langue basque où aucun mot ancien ne peut commencer par $r$, la voyelle prothétique n'étant pas cependant ici $a$-comme en galicien, en asturien et en gascon, mais au contraire $e_{-}$, dans errege < regem, erripa < ripam, etc.

Nous présentons par ordre alphabétique une liste non-exhaustive de toponymes qui, on l'a dit, se répètent ou semblent se répéter en Galice et en Pays Basque, voire parfois en Béarn et en Aragon. Nous utilisons presque toujours les formes anciennes recueillies, il y a plus de cinquante ans, par Víctor Olano Silva, c'est-à-dire environ mille noms médiévaux représentant à eux seuls la quasi totalité des formes anciennes connues, ce qui est malheureusement peu eu égard à la quantité considérable de toponymes que compte un pays comme la Galice.

1. Aldariz/Alderiz/Alderís : dans la province de La Corogne, on trouve un village appelé Alderis et un autre Alderiz. Dans la province de Pontevedra un hameau est également appelé Aldariz; en galicien l'alternance -riz/-rís est due à un phénomène dialectal appelé "seseo", c'est-à-dire un défaut qui consiste à prononcer en espagnol, en l'occurrence ici en galicien, les $c$ comme les $s$, ce qui explique les doublets toponymiques galiciens Esmoriz/Baldariz (paroxytons) et Esmoris/Baldarís (oxytons), etc. En Navarre, dans la vallée d'Ezkabarte (bailliage de Pampelune), Altadil! signale une maison seigneuriale appelée Alderiz. Ce toponyme basco-galicien est d'origine germanique : Aldericus (cf. Marie-Thérèse Morlet).

2. Ames: la forme ancienne du toponyme béarnais Ogenne (canton de Navarrenx) est Ogene, XI' siècle, Oiena, XIII siècle, Oyene, 1385. Michel Grosclaude considère qu'il s'agit du basque oihan, "forêt", hypothèse qui semble faire l'unanimité. Dans la province de La Corogne, on trouve une paroisse appelée Santo Tomás de Ames (municipalité de Negreira), autrefois Sci. thome de oianes, 1228, l'évolution ayant vraisemblablement été oianes $>*_{o}$ anes (confusion avec l'article masculin galicien $o$ ) $>*^{*}$ anes $>$ ames. On remarquera la terminaison inexpliquée -es dans oianes qui semble correspondre à un ethnique. La signification de ce nom galicien n'est pas connue. 
3. Anós et Ois: d'après G. Rolhfs' ${ }^{13}$ le toponyme Agnos, Anhos, 1364 (canton d'Oloron-Sainte-Marie) est issu du gentilice Annius, mais en revanche les nom des villages appelés Anos, 1243 (canton de Morlàas) et Anoz (vallée d'Ezkabarte, Navarre) seraient peut-être issus du cognomen Andus + suffixe aquitanique *ossu, le changement de $n d$ à $n$ étant normal en gascon. En Navarre, ce changement ne peut cependant être dû, comme le rappelle Luis Michelena ${ }^{14}$, qu'à l'influence du parler aragonais : *Garindoain > Garinoain, Garinnoain en 1072 (Olite, Navarre). Dans la province galicienne de La Corogne, on trouve un village appelée Anós, accentué sur la dernière syllabe. Le nom n'est expliqué par aucun auteur et il ne semble pas qu'on en connaisse la forme ancienne. En galicien, le groupe $n y$ passe normalement à $-\tilde{n}$-, le gentilice Annius, et sa variante Annianus, étant ainsi à l'origine, comme le rappelle Joseph M. Piel's, de plusieurs noms tels que Añá (La Corogne), probablement Añón (La Corogne), Vilañán (Lugo), Añobres/Añobre (La Corogne), etc., ce dernier étant un quasi homonyme du toponyme navarrais Añorbe signalé par J. Gifford, mais il s'agit manifestement d'un paronyme. Grâce aux lois phonétiques du galicien nous pouvons restituer l'étymon qui serait peut-être à la base du nom galicien Anós, c'est-à-dire probablement (avec une terminaison en $o$-, -onis) *annonis $>$ *annones (avec évolution romane et normale du - $i$ - dans -onis qui passe-ones) $>{ }^{*}$ anoes $>{ }^{*}$ anois $>$ anós. En revanche, on est sûr de l'origine du toponyme Ois (Santiago de Ois, village de La Corogne), autrefois in loco adones, 910 dont l'évolution n'a pu être manifestement que : adones $>{ }^{*}$ aoes $>{ }^{*}$ a ois (confusion avec l'article galicien féminin a) > ois, ce dernier étant peut-être en rapport avec les toponymes Oiz, en basque Oitz (montagne de Biscaye et village de Navarre, Malerreka, Pampelune) et peutêtre avec celui de Aoiz, en basque Agoitz, Aoitz (bailliage de Sangüesa). Le toponyme galicien Adones doit être comparé avec le nom de personne Aio, génitif Aionis, retrouvé par Alfonso Irigoyen' ${ }^{16}$ dans une inscription romaine d'Estrémadure : Camilius/Arrus/Aionis f./Clun. an./LXX hic. Ce nom serait à l'origine, d'après cet auteur, du toponyme navarrais Aoiz (chute du [j], du $-n$ intervocalique et équivalence $s$ latine $=z$ fricative dorso-alvéolaire basque d'après Luis Michelena, neutralisée en basque en position finale en tant qu'affriquée $-z>-t z$ ), le nom étant prononcé par les bascophones Agoitz avec - $g$ - antiyod. Alfonso Irigoyen cite également la variante romanisée et médiévale de ce nom navarrais : noble don Per Ahones, 1320, très proche de notre Adones galicien et également médiéval.

4. Aranga: la signification du nom de ce village galicien de La Corogne, situé dans une vallée entourée de montagnes d'une altitude moyenne de $700 \mathrm{~m}$, n'est pas connue. Le nom apparait au moyen âge dans une bulle confirmant les possessions de l'Église de Compostelle : et Superato et Aranga et Montejaurino quomodo diuidit cum Parrega de Prutiis et Bisanquis, 1178. L'élément -anga se trouve, rappelle Luis Michelena ${ }^{17}$, dans les patronymes basques Estanga, Uranga, Uzkanga, etc. et n'a jamais été expliqué de manière satisfaisante. En outre, deux autres villages galiciens de la province de La Corogne s'appellent respectivement Arangas et Laranga. Le nom apparaît également fréquemment dans les Asturies : Arango (municipalité de Pravia), Las Arangas (municipalité de Grado, en asturien Grau), Arangas (municipalité de Cabrales), Aranguin (Pravia), etc. et en Navarre, on a le village appelé Elcóaz Arangoz dans un document ${ }^{18}$ du XV siècle (vallée d'Urraúl, bailliage de Sangüiesa), aujourd'hui manifestement Arangozki. Ces noms galaïco-basco-asturs sont peut-être à mettre en rapport avec un hypothétique vocable 
pré-latin *ara apparenté probablement avec le basque (h)aran, ara (n)-, "vallée". Les deux villages galiciens appelés Aranza (Pontevedra et Lugo), malgré une surprenante homonymie, n'ont rien à voir avec le nom navarrais Arantza (village de Cinco-Villas, bailliage de Pampelune) et probablement non plus avec le patronyme Aranza cité par Michelena étant donné que la forme navarraise Arantza est récente, I'ancienne forme du nom de ce village étant Aranaz, 1280, Aranaz de las' cinco villas de Lerin, 1644. Les formes anciennes des noms des villages galiciens appelés Aranza ne sont pas, sauf erreur, connues et le nom est inexpliqué. Voici la liste des noms de villages et de paroisses galiciens où apparaît la forme aran et qui font difficulté, l'absence, pour la plupart d'entre eux, des formes anciennes empêchant toute explication. Province de Lugo : Arán, Fontarán, Arangón, Malvarán, San Pedro de Arante, S. P. de Alanti, 935 et Aralde; province de Pontevedra : Aralde (autre), Aranés, Arantey et San Pedro de Arentey; province de La Corogne : Arán (autre), Fonte Arán, Araño (cf. Arano, bailliage de Pampelune) et San Vicente de Arantón, aranton, 830, nom qui rappelle la ville vasconne d'Alantone cité dans l'Itinéraire d'Antonin et située à l'époque entre Aracaeli ou Araceli (Huarte-Arakil) et Pampelune. On aurait tendance de nos jours à identifier ce toponyme vascon Alantone avec l'actuel village navarrais appelé Atondo (Cendea d'Iza, bailliage de Pampelune). L'équivalence galaïcovasconne Alanti, 935 (Arante, Lugo) $=$ Aranton, 830 (Arantón, La Corogne) $=$ It. Ant. Alantone (prob. Atondo, Navarre) doit être notée.

5. Arga/Argá : la signification du nom de la rivière navarraise appelée Arga est inconnue. Un village de la province de Lugo s'appelle Argá (ici toutefois il s'agit d'un oxyton), une rivière et une montagne de cette province étant également connues sous le nom de Argán qui, d'après Azkue, signifie en biscaien "terrain pierreux". En théorie, Argán pourrait s'expliquer par le basque arr (i) + gain $>$ argain $>$ argan, "hauteur rocheuse" comparable au toponyme labourdin *aitz + gain > azkain. Il est difficile de savoir quelle peut être la relation entre ces toponymes car les formes anciennes des noms galiciens ne sont pas, sauf erreur, connues. Joan Coromines ${ }^{19}$ cite également un arga pré-romain, sans en donner le sens, qu'il suppose d'origine celtique ou ibérique.

6. Argote: le toponyme alavais Argote se retrouve en Galice où il est porté par un village de La Corogne.

7. Arinez/Ariñez/Arines : le nom de village alavais Arinez/Ariñez, autrefois Ariniz, cité par José Miguel de Barandiarán, semble être identique à celui porté par une paroisse et une rivière galiciennes : Arínes (La Corogne).

8. Arros: dans la province de La Corogne, un village s'appelle Arros (paroxyton, sauf erreur). Il ressemble aux toponymes bas-navarrais et béarnais Arros, le bas-navarrais étant prononcé en basque Arrotze, d'après Lhande. G. Rohlfs cite, entre outre, Arrós (Alto Pallars, province de Lérida) et Arrós (Val d'Aran, province de Lérida). En ce qui concerne le toponyme galicien, il pourrait être issu, mais ce n'est pas sûr, du "cognomen Arro attesté dans des inscriptions d'Espagne et de Lusitanie (CIL, II, 2735 et 5610)", selon G. Rohlfs ${ }^{20}$.

9. Asma: de nos jours un village situé au pied d'une montagne de $838 \mathrm{~m}$. et un fleuve de Lugo sont appelés Masma, autrefois le fleuve et toute la région étant appelés : in nasue, 747 ? (avec $u=b$, c'est-à-dire nasue $=$ nasbe) ; Asma, 832 ; territorio Assue (=Assbe) et Asme, 871;Asua et Asma, 897 ; Asma, 1154, 1158 ; 
ambas Asmas, 1173 ; aujourd'hui Masma. Ce nom galicien rappelle étrangement celui de la vallée d'Aspe (Béarn autrefois $A s p a, 1017$ ), celui du village navarrais appelé $A z p a$ (vallée d'Egüés, Sangüesa, autrefois $A s p a, 1197$ ) et les noms de villages pyrénéens Asme (Basse-Navarre, autrefois Azpe, 1413, azme, 1481), Aspet (H-G.), Aspin (H-Pyr.) ainsi que celui d'un village aragonais, aujourd'hui abandonné, appelé Aspe (autrefois Aspe et $A s p a, \mathrm{XJ}^{e}$ siècle) et pour lesquels la quasi totalité des auteurs admet une origine pré-romane : aitz + -pe, "en bas de la hauteur rocheuse". Le toponyme galicien Assue, Asua, Asma, Asme doit probablement signifier la même chose, car sinon il est inexplicable. Un autre village et une rivière de la province de Lugo ont pour nom Asma, le village étant situé au pied d'une montagne de $760 \mathrm{~m}$. Dans cette même province, un village situé au pied d'une montagne de $735 \mathrm{~m}$. est appelé $A s p a i$, mais les formes anciennes de ce nom ne sont pas connues, sauf erreur.

10. Baiona (graphie galicienne et officielle) / Bayona (graphie espagnole) / Baiona (aujourd'hui en français Bayonne). Il s'agit bien évidemment du cas le plus célèbre. Alfonso Irigoyen n'acceptait pas l'hypothèse de Jean-Baptiste Orpustan selon laquelle dans le nom labourdin Baiona on a probablement affaire à l'élément pré-indo-européen bai-, "cours d'eau" (cf. supra) car, écrivait-il, "en relación con el caso del de Galicia nos haría entrar en elucubraciones complejas de muy dificil solución" ${ }^{21}$. Il ne prenait pas en compte le fait que l'ancien et véritable nom de ce village galicien était jusqu'au XII e siècle Erizana, époque à laquelle il changea de nom pour s'appeler Baiona. D'après Eugène Goyheneche, “il s'agit sans doute d'un repeuplement, d'une ville neuve, fondée au même endroit qu'Erizana (terme ancien) dépeuplée par les Invasions" étant donné qu'"on donnait à ces villes, souvent un nom déjà porté par une autre ville, plus célèbre ou plus ancienne. Il n'est donc pas impossible qu'en 1130 Alphonse VIII ait donné ce nom à Bayona de Galice, en se souvenant de notre Bayonne" ${ }^{22}$. Le nom Erizana existe encore de nos jours : Cabo Erizana, "Cap d'Erizana" (Pontevedra), nom considéré par certains comme étant d'origine pré-indo-européenne. La forme ancienne est inconnue, mais il est probable qu'à l'initiale l'évolution a été : Eri- $<*^{*} l l i$-, cet élément ${ }^{*} I l i$ - se retrouvant sous la forme Iri-dans le nom du village galicien de Lugo appelé Orizón, autrefois Irizon, 1032. Régis de Saint-Jouan, dans son dictionnaire, cite les noms de maisons (L')Irisson et (L')Irissoo, 1385 (Bellocq, bailliages de Rivière-Gave et de Pau). D. Ramón Menéndez Pidal ${ }^{23}$ rappelle que la ville galicienne actuellement appelée Padrón (La Corogne) s'appelait dans l'Antiquité Iria Flavia, nom inexpliqué et qu'on a voulu rapprocher du vocable ili-/iri-présent dans les noms Illiberris (Elvira à côté de Grenade, Andalousie), Illiberis (Pline), Eliberri (Mela), Ilibirris (Strabon), aujourd'hui Elne (Pyr.- Or.), etc.

11. Bardaos: dans la province galicienne de Pontevedra, on trouve la paroisse de Santa Maria de Bardaos (municipalité de Tordoia), autrefois Bardanis, 1228. Ce nom se rapproche curieusement de celui des Bardenas, en Navarre, autrefois, bardenas, 1155, Bardena, 1212, dont on n'a pas trouvé d'explication satisfaisante. Dans la province de La Corogne, on trouve également un village appelé Bardullas qui rappelle le nom de peuple Vardulli ou Varduli - ancêtres grosso modo des Guipuscoans actuels - cité par Mela (Livre, III, 15) et Pline (Histoire Naturelle, Livre III, 27). Julio Caro Baroja ${ }^{21}$ rappelle que dans l'Antiquité, un peuple de Lusitanie était également appelé par le même nom : Bardili (Pline, Histoire Naturelle, Livre IV, 118). 
12. Beade: le village de Santa María de Beade (province d'Orense) s'appelait autrefois Sanctae Mariae de Vilate, 1112 ; en galicien la chute de la latérale, la sonorisation du - $t$ - intervocalique et l'instabilité, courante en galicien, des voyelles $i / e$ expliquant la forme moderne Beade. Ce nom semble se retrouver en Pays Basque, comme le signale J. Gifford, dans le nom du col navarrais de Velate (orthographié en basque Belate) que l'on considère d'ordinaire comme étant issu du basque bele, "corbeau", en composition bela-, et ate, "porte, passage", soit "porte, passage des corbeaux". Il s'avère problématique de penser que la forme galicienne Vilate puisse s'expliquer par le basque. Il faut également citer le peuple aquitain des Vellates cité par Pline (Histoire Naturelle, Livre III, 33). Il s'agit peut-être d'une coïncidence. Quoi qu'il en soit, affirmer que le toponyme navarrais Bélate est un nom basque parait prématuré.

13. Beariz: en 1992, un érudit local ${ }^{25}$ a découvert dans un cartulaire conservé dans le monastère cistercien d'Oseira (municipalité de Cea, Orense) les formes anciennes, du nom de la paroisse galicienne appelée Santa María de Beariz (municipalité de Beariz, orense). On sait à présent que ce nom galicien est un toponyme d'origine germanique, les formes anciennes étant : viarici, 1034, uiarici, uiariz, 1053, veariz, 1220. Le nom est issu de l'anthroponyme germanique, attesté en Galice comme le rappelle Ana Isabel Boullón Agrelo, Viaricus, romanisé par la suite en Viarigo. Dans un article paru récemment dans Fontes ${ }^{26}$, nous avons émis l'hypothèse selon laquelle le toponyme labourdin Biarritz, autrefois beariz, 1150 , bearriz, 1150 , bearidz, 1186, bearritz, 1249, bearys, 1344, bearitz, 1511 , pourrait peut-être avoir la même origine que ce nom galicien, d'autres toponymes basques d'origine germanique étant attestés en Navarre. Nous ne reviendrons pas sur l'argumentation développée dans cet article auquel il suffira de se reporter. Nous rappellerons simplement qu'il existe une difficulté. Pour accepter notre hypothèse, il faudrait être certain, ce qui n'est pas le cas, que dans les formes anciennes du nom Biarritz, c'est bien la vibrante faible intervocalique $-r$ qui est étymologique et non l'inverse. Si c'était le cas, l'équivalence veariz/beariz galicien beariz/bearriz labourdin serait envisageable, car le parallélisme est frappant. Cependant, il est peut-être préférable de considérer la vibrante forte comme étant étymologique. En effet, lorsqu'on a affaire dans les formes médiévales d'un nom à l'alternance $-r-/-r r$-, c'est presque toujours la forme avec vibrante faible qui est fautive et il faut reconnaître que dans le nom Biarritz la forme avec $-r r$ forte est constante depuis les débuts. Il existe deux autres paroisses galiciennes portant le même nom : San Martín de Beariz (municipalité de San Amaro, Orense) et Santa Magdalena de Baariz, 1392 (municipalité de Paradela, Lugo).

14. Beascós: dans la province de Lugo, on trouve le village de Santa Marina de Beascós (municipalité de Carballeda), autrefois Sancto Martino de Velascones, 1175. Ce nom rappelle le nom aquitain, attesté à l'époque romaine, Belexconis (et sa variante Belasconis citée par Michelena et Irigaray) et qui pourrait être d'après ces deux auteurs à l'origine du nom Briscous, en basque Beskoitze, autrefois Berascoiz(co), "(de) Briscous" d'après Lissarague qui en était natif. La chute du - $n$ - intervocalique et le passage de $-l$-intervocalique à $-r$ - sont des phénomènes courants en basque. Jean-Baptiste Orpustan ${ }^{27}$ propose également une autre hypothèse. En ce qui concerne le toponyme de Lugo, il est certain qu'il provient d'un plus ancien *Velasconis, en galicien l'évolution romane de la voyelle $i$ à $e$ dans la terminaison -nis étant tout à fait normale et régulière, les exemples qui le montrent étant nombreux, et par conséquent, il est sûr qu'en Galice on a eu une forme 
plus ancienne Velasconis ou Belasconis qui a normalement évolué en Velascones puis en Beascós : changement de $v$ en $b$ et amuïssement automatique de la latérale ainsi que de la nasale intervocaliques.

15. Beasque: nous avons trouvé dans la municipalité galicienne de Ponteareas (Pontevedra), un hameau appelé Beasque (81 habitants en 1980), nom qui rappelle le toponyme bas-navarrais Béhasque. On ne connaît pas la forme ancienne du nom galaïque, mais les lois phonétiques du galicien permettent de la reconstituer. Cette forme ne peut être manifestement que Velasco puisqu'en galicien $v$ passe à $b$, la latérale intervocalique s'efface et -o final s'affaiblit en -e (comme dans le toponyme galaïque actuel Grondaisque < Gandaisco, 1128); d'où en galicien Velasco > Beasque. Ce nom viendrait appuyer l'hypothèse selon laquelle le nom navarrais Béhasque est issu lui aussi, comme le pense également JeanBaptiste Orpustan ${ }^{2 s}$, de l'anthroponyme Velasco ; avec cependant un suffixe d'appartenance -anum. Jean-Baptiste Orpustan cite également une autre hypothèse purement toponymique. Toutefois, bien que le résultat soit apparemment le même dans Beasque/Béhasque, l'évolution qui a été celle de ces deux noms est différente. En basque le passage de $v$ à $b$ existe, mais la chute de la latérale n'est pas automatique comme en galicien. L'évolution a donc été : Velasco + -anum $>$ Belask $(o)+$-ain $>$ Berask $(o)+$-ain (avec $-l->-r-$ ) $>$ Behask $(o)+$-ain avec vibrante faible qui s'atténue entre voyelles jusqu'à $h$ (cf. uralde $>$ uhalde, etc.). A la finale non plus, l'évolution n'a pas été la même puisque c'est l'accentuation gasconne qui explique le passage Beháskain > Behásken > Behásque, la forme basque étant Behaskane.

16. Beleia/Velegia/Pons Vellegia : I'Itinéraire d'Antonin cite un endroit galicien appelé Pons Vellegia (aujourd'hui Ponte Vea, La Corogne). Le nom paraît devoir être rapproché de celui de la ville des Caristes appelée dans l'Antiquité Ouéleia/Beleia ou Velegia (aujourd'hui Iruña, Alava).

17. Bestar : l'anthroponyme médiéval Belastar, San Millán, 952, d'origine basque d'après Gonzalo Diez Melcón ${ }^{29}$ et dans lequel on croit voir, comme le rappelle Luis Michelena ${ }^{30}$, le suffixe basque - $(t) a r$ semble se retrouver également dans le nom de la paroisse galicienne de Santa María de Bestar (municipalité de Cospeito, Lugo), autrefois Sancta Maria de Valestar, 897, Belestari, 1062. On le rencontre probablement aussi dans le nom de la paroisse également luquoise de San Pedro de Besteiro; autrefois S. P. de Baestarios, $1164<{ }^{*}$ Balestairos $<{ }^{*}$ Balestarios, en galicien $i$ semi-voyelle dans le groupe $r y$ reculant jusqu'à la syllabe précédente : $-r y$ - $>-i r$ - A cette époque la chute de la latérale intervocalique avait déjà eu lieu en galicien.

18. Bioron/Biurraran/Biurrun: d'après J. Gifford la rivière galicienne appelée de nos jours Luyña ou Naviego (Orense) était appelée autrefois Bioron, 11 12, le nom n'étant manifestement ni d'origine celtique ni latine. Dans le village navarrais de Lesaka (bailliage de Pampelune), il existe une rivière sinueuse appelée tântot Uarte, tantôt Biurgarai et un quartier connu sous le nom de Biurrarango auzua. J. Gifford compare le nom de cette rivière galicienne avec celui du village navarrais appelé Biurrun (Valdizarbe, Pampelune), autrefois Bihurrun, 1272, Biurun, 1345, issu du basque bi (h)ur, "tordu" et -un, "lieu, endroit". Le nom galicien n'est pas expliqué. 
19. Buño, la montagne de $738 \mathrm{~m}$. surplombant le village galicien d'Aranga (La Corogne) est appelé Buño, nom que porte également un village proche de la ville de La Corogne. Ces deux noms doivent être vraisemblablement rapprochés du radical oronymique pré-latin bun-" ${ }^{3 !}$, "hauteur" présent dans le toponyme bas-navarrais Bunus, bunos, 1304 (suivi ici du suffixe aquitanique -os) et également dans le lexique basque actuel sous les variantes nasalisées muno, munho, muño, "colline".

20. Burés et Bares : dans la province de La Corogne - non loin du cap Finistère, autrefois appelé Promonterium Nerio, du nom de la tribu galaïque des Nerio appartenant d'après Pomponius Mela à la vaste confédération des Artabri dans un endroit montagneux situé en bord de mer, on trouve le village de Burés dont le nom rappelle curieusement le toponyme béarnais Buros dans lequel il faut peut-être voir le thème pré-latin *bur-32, "hauteur" (cf. basque buru, "tête") variante du radical bun- déjà cité. Dans la province de Pontevedra, on trouve également deux montagnes appelées respectivement Buroz et Burizo. A l'extrémité nord-ouest de la province de La Corogne, au milieu d'un littoral extrêmement découpé, une bande de terre atteignant, dans sa partie la plus élevée, une hauteur de $210 \mathrm{~m}$., s'avance dans la mer sur une distance d'environ $5 \mathrm{~km}$. L'endroit est appelé de nos jours Punta da Estaca de Bares. Situé presque à la limite de la pointe de Bares, se trouve le village de Vila de Bares appelé dans l'Antiquité Burum (Ptolémée, II, 6). Ce toponyme galicien de l'époque romaine contient manifestement le vocable pré-indo-européen (et aujourd'hui basque) buru, "extrémité, cime, cap, tête". L'Itinéraire d'Antonin cite un lieu galicien appelé Burbida situé probablement, d'après D. Ramón Menéndez Pidal, à côté de l'actuelle paroisse de Santiago de Borbén (Pontevedra). Toujours d'après D. M. Pidal, il s'agirait de buru, "cima" et bide, "camino, camino de la montaña"33. Joan Coromines ${ }^{34}$ pense également qu'il s'agit de buru-bide.

21. Caboy et Caaveiro: dans la province de Lugo, on trouve la paroisse de San Martín de Caboi (municipalité d'Otero de Rei) appelée autrefois Calabogi, 959, Cabogi, 1127, Caabogi, 1231 avec chute de la latérale intervocalique. L'élément initial cala- de ce nom galicien est identique à celui qu'on trouve, entre autres, dans le toponyme attesté à l'époque romaine Calagurris (Calahorra), ce dernier étant le nom d'une ville considérée à l'époque comme vasconne. Dans la province de La Corogne, on trouve également le village de Caaveiro, autrefois sci. iohannis de calauario, 936 ?, scm. Johannem de Calaueyro, 1199. Toujours dans cette province, une plage et une pointe rocheuse s'avançant dans la mer s'appellent Caveiro. La forme ancienne de ce nom n'est pas connue, mais ici aussi il est probable que le cainitial est issu d'un plus ancien *cala-, tout comme dans le nom du village luquois appelé Caamonde, issu probablement d'un ancien *calamundi.

22. Ceanuri : Alfonso Irigoyen ${ }^{35}$ avait rapproché le nom de la ville biscaïenne de Ceanuri, dont la forme ancienne n'est pas connue, comme le rappelait Luis Michelena, des nombreux noms de lieux galiciens appelés Ceán, dont celui d'un village de la province de Pontevedra et d'une montagne de La Corogne. Le sens de Ceanuri serait donc "ville de Ceán". Luis Michelena y voyait un *Azenar-uri avec dissimilation de la vibrante et chute en basque du $n$ intervocalique : "ville d'Aznar" 36.

23. Cedeira/Cettaria : le village de Cedeira est situé en bord de mer, au nord de La Corogne. A l'époque romaine, il était appelé Cettaria $(C=K)^{37}$. L'évolution Cettaria $>$ Cedeira est normale en galicien. Une montagne de La Corogne de 600 
m. s'appelle également Cedeira. La forme Cettaria doit peut-être être rapprochée du nom du village labourdin appelé Guéthary, autrefois Cattarie, 1193 d'après Paul Raymond, et aussi sans doute du Getaria guipuscoan. En ce qui concerne le village labourdin de Guéthary, on y a retrouvé des traces d'un camp romain, mais la signification exacte du nom n'est pas assurée.

24. Deba/Deva : cet hydronyme est d'origine celtique. Le fleuve guipuscoan appelé Deba (c'est aussi le nom d'une ville guipuscoane) séparait dans l'Antiquité le peuple des Caristes (ancêtres grosso modo des Biscaïens actuels) de celui de Vardules (ancêtres grosso modo des Guipuscoans actuels). Le nom est également celui d'un fleuve cantabro-asturien puisqu'il sert aujourd'hui de frontière aux communautés autonomes des Asturies et de Cantabrie, ce fleuve étant situé dans l'Antiquité en territoire cantabre. En Galice, ce nom apparaît écrit tantôt Deva, tantôt Deba : c'est celui d'une rivière traversant les provinces d'Orense et de Pontevedra et c'est également le nom d'une paroisse de Pontevedra.

25. Ego : il y avait chez les Galaïques une ville appelée Egovarri (Pline, I, 227, 7). Ce nom est en rapport, comme on le verra plus loin, avec le nom du fleuve appelé Eo qui sépare aujourd'hui les Asturies de la Galice. Dans l'Antiquité, ce fleuve était situé à l'intérieur du territoire galaïque qui s'étendait jusqu'aux chaînes de montagnes, actuellement en territoire asturien, appelées Sierra del Rañadoiro et Sierra de Uría où commençait alors le pays des Astures transmontani, c'est-à-dire grosso modo les Asturiens actuels. Bien que les forme anciennes et médiévales de l'hydronyme Eo soient, d'après Víctor Olano Silva et à tort comme on le verra par la suite, Yube, 775, euve, eube, 925, eunio, 1128, Oue, XII ${ }^{\circ}$ siècle, on peut affirmer que le nom Egovarri est à l'origine de l'hydronyme Eo pour une simple et unique raison: les actuels habitants des rives de ce fleuve aujourd'hui galaïco-astur, c'est-à-dire principalement ceux des villages galiciens de Ribadeo et d'Obe et ceux des villages asturiens de Vegadeo et de Castropol, sont encore de nos jours désignés par le terme générique Egovarros ${ }^{38}$. Le nom Egovarri cité par Pline rappelle à l'initiale celui de l'actuelle rivière basque appelée Ego, autrefois Ego, $1335^{39}$, cours d'eau passant entre les localités d'Ermua (Biscaye) et d'Eibar (Guipuscoa) et également celui de la rivière navarraise appelée Ega. Au début du XIX ${ }^{e}$ siècle, W. von Humboldt avait analysé ce nom galaïque de l'époque romaine à partir du basque : ego-varri, "nouvelle demeure". On ne peut lui en tenir rigueur car à son époque, on ne connaissait pas les formes anciennes du nom de ce fleuve. En fait, c'est la connaissance et l'examen des formes médiévales de cet hydronyme qui nous ont permis de comprendre et d'analyser la forme Egovarri citée par Pline : le - $i$ final étant la déclinaison latine (Humboldt n'avait pas compris qu'i] s'agissait de la déclinaison latine et c'est pourquoi il avait pensé au biscaïen -barri, "neuf, nouveau"), ce qu'il faut en réalité analyser, c'est la forme Egovarr ou Egobarr. Les pièces du puzzle s'emboîtent à présent toutes les unes dans les autres. Ainsi, ce nom galicien de l'Antiquité doit s'analyser egob-arr, avec le suffixe vascoïde -arr et sa variante -tarr qui servent encore de nos jours en basque à indiquer l'origine : kanboarr, "originaire de Cambo", Baigorriarr, "originaire de Baigorry", Angeluarr, "originaire d'Anglet", Lapurtarr, "Labourdin", etc. Le nom Egobarr doit signifier "originaire, habitant de Egobe". On comprend à présent les formes médiévales galiciennes Yube, Eube, Euve, etc. où on a eu affaire en galicien à la chute du -g-intervocalique (phénomène de phonétique galicienne); d'où Egobe $>$ Eube $>$ oue $>O b e$, c'est-à-dire aujourd'hui le nom d'un village galicien situé à côté de l'embouchu- 
re du fleuve Eo. Víctor Olano Silva s'était trompé : Eube, Euve, Oue, etc. ne sont les formes anciennes du nom de ce fleuve, comme il le croyait, l'erreur s'étant perpétuée chez les quelques rares auteurs qui le mentionnent, mais au contraire les formes médiévales du nom de l'actuel village galicien d'Obe. Et ce village s'appelait à l'époque de Pline Egobe, ses habitants étant appelés Egobarr. Le nom a par la suite été latinisé en Egovarri, forme qui existe encore actuellement puisque, on l'a vu, les habitants de la région sont appelés Egovarros. Et que signifie le nom Egobe, aujourd'hui Obe? Il signifie tout simplement Ego-be, "au pied de l'Ego", c'est-à-dire "à l'embouchure du fleuve Ego", aujourd'hui appelé Eo (chute normale en galicien du - $g$-intervocalique), le suffixe -be/-pe, encore utilisé de nos jours en basque, signifiant "sous, au pied de, en bas de". Et Ego, on l'a dit, est le même nom que celui porté encore de nos jours par une rivière basque. On peut donc dire qu'à l'époque romaine, il y avait parmi les Galaïques des peuples qui utilisaient les suffixe -arr et -be (cf. $\S 10$. Asma, supra) indiquant l'un l'origine et l'autre la position, suffixes qui se retrouvent encore de nos jours en basque avec la même signification. Cela nous ouvre manifestement des perspectives sur la Galice pré-romaine.

26. Igón/Igon : il est désormais établi que le toponyme béarnais Igon est d'origine pré-indo-européenne ${ }^{40}$. Un village de la province de Lugo s'appelle Igón. Il faudrait en connaître la forme ancienne pour savoir de quoi il s'agit exactement.

27. Igueldo/Gueldo: une montagne galicienne s'appelle Gueldo (La Corogne), nom qui rappelle curieusement la montagne de Saint-Sébastien appelée Igueldo.

28. Izaro/Ézaro: d'après Azkue, l'île située dans la baie de Bermeo (Biscaye) a pour nom Izaro, mot qui pourrait peut-être signifier, toujours d'après cet auteur, "île" en général. Juan Antonio de Moguel, dans son roman intitulé Peru Abarca, dit que le mot signifie "círculo de mar". On retrouve manifestement le même nom dans la province de La Corogne où un village situé en bord de mer s'appelle Ézaro, l'alternance $i$-/e- demandant cependant à être expliquée, à moins que le biscaïen Izaro ne soit au contraire issu d'un ancien *ezaro, quoique la langue galicienne connaisse l'alternance vocalique $i / e$, principalement à l'intérieur des mots : único $>$ úneco, etc. en asturien cette alternance vocalique pouvant se rencontrer également devant une sifflante : historia > hestoria, hestóricu, etc. Le village d'Ézaro, situé à côté du Cap Finistère, se trouve au pied d'une petite montagne qui tombe à pic dans la mer. Le village est situé à l'intérieur d'une toute petite baie en forme de demi-cercle. Le fleuve appelé Xallas ou Ézaro, situé pratiquement au dessus du village, se jetait autrefois directement dans la mer depuis une hauteur de $300 \mathrm{~m}$., ce qui en faisait un spectacle presque unique en Europe. Plus tard, l'entreprise espagnole FENOSA supprima cette chute d'eau. Aujourd'hui, le fleuve se jette dans la mer le long de canalisations qui fournissent de l'énergie électrique. L'équivalence présumée Izaro biscaïen = Ezaro galicien avait déjà été remarquée et soulignée par Carlos Jordán Cólera ${ }^{41}$, qui compare également, entre autres, les hydronymes Isara et Ésera, ce toponyme galaïcobasque n'étant sans doute pas, comme le pensaient Azkue et Moguel, un mot basque, mais plutôt un mot d'origine inconnue où il faudrait voir la racine, vraisemblablement hydronymique, ${ }^{*} i z-$ ou ${ }^{*} e z-$, Carlos Jordán Cólera allant jusqu’à proposer une racine hydronymique paléo-européenne *eis/*is, "se mouvoir avec rapidité, violemment". Dans le nom Iso (nom d'une rivière de province de La 
Corogne), il semblerait qu'il y ait également cette racine. Un village de Lugo s'appelle Izá (oxyton). Le nom semblerait être issu de l'anthroponyme celte Ícilus, son dérivé étant Iccianus ${ }^{42}$. Ce nom galicien n'a très probablement rien à voir le toponyme navarrais Iza, autrefois Hiç̧a, Hiiza, 1124, issu manifestement du basque $i(h) i+-t z a$, "jonchaie".

29. Langara/Láncara: un quartier du village alavais d'Arratzu-Ubarrundia est appelé Langara, nom qui se retrouve dans celui d'un village, d'une montagne et d'une vallée de la province de Lugo appelés tous les trois Láncara (proparoxyton), au moyen âge également lancara (cf. $G G R G$ ), en basque la sonorisation de l'occlusive après $n$ étant normale : *Lankara $>$ Langara. Le village galicien est connu car il s'agit du village natal du père de Fidel Castro. D'après Moralejo Lasso $^{43}$, qui cite Johannes Hubschmid, un suffixe -ara atone semble se rencontrer dans plusieurs toponymes : Bracara (aujourd'hui Braga), Lózara (Lugo), Tállara (La Corogne), Tábara (Zamora), etc. et dans les vocables cáscara, gándara, etc. Luis Michelena cite également le toponyme et patronyme basques Langarika (quartier d'Iruraitz-Gauna, Alava) dans lequel le suffixe -ika, très abondant en Biscaye et en Alava serait d'origine celtique. Curieusement, ce suffixe, dont l'origine celte semble faire l'unanimité, n'est presque pas représenté dans la toponymie galicienne. Un seul village situé en bord de mer semble le porter : Malpica de Bergantiños (La Corogne) qui doit être cependant une formation tautologique : *mal, "hauteur" et pico/pica > Malpica, le village étant situé sur une presqu'île en forme de colline, une montagne de Lugo et une autre d'Orense s'appelant également Malpique < *Malpico. Le suffixe -ica semble figurer dans le nom de quelques hameaux : Chafarica (Lugo) et Arcabrica (Orense) et peut-être dans le nom du cap appelé Cabo de Nariga ( $<*$ Narica) ainsi que sans doute dans les endroits connus sous les noms de Punta de Pelica et Monte de Subica (tous situés dans province de La Corogne). Dans ces noms galiciens, il faut noter l'absence de sonorisation de l'occlusive sourde intervocalique. En théorie pourtant, ces dernières devraient toutes se sonoriser de façon systématique en galicien. L'évolution du nom du village de Lugo appelé Parga est à ce propos significative : Aparraca ou Aparraqua (époque romaine, cf. tribu galaïque des Aparraques) > Parraqua $>$ Párrega, 1178 et Párraga (maison noble et seigneuriale) > Parga (nom actuel). Ainsi, on aurait dû avoir : -ika > -iga comme cela est le cas dans le nom Nariga, issu manifestement de *Narica. Mais curieusement cela n'est pas le cas pour les autres: Subica, Pelica, etc.

30. Larrate : il existe en Navarre deux lieux appelés Larrate. Le premier est le nom d'un lieu-dit qui se trouve dans la municipalité de Fitero, Larrate de Vinuelas, 1254, aujourd'hui La Rate (municipalité située dans l'extrème sud de la Navarre et appartenant au bailliage de Tudela) et le second est le nom d'une colline située à côté du village de Carcastillo (bailliage de Tudela, la rat, 1319 et la Rate, 1844). Plusieurs auteurs, dont, entre autres, Jesús Arzamendi, Ricardo Ciérbide $^{44}$ et Luis Michelena, y voient probablement un nom basque : "porte, passage de la lande" ou "lieu de landes" si le suffixe -ate est ici une variante du suffixe locatif -eta. Erlantz Urtasun ${ }^{45}$ pense que ce nom pourrait être une preuve de l'emploi autrefois de la langue basque dans cette région. Cependant, J. Giffford fait état d'un toponyme galicien Larrate en 853 (province d'Orense). Considérer que ce nom galicien médiéval est un basquisme s'avère délicat. Par conséquent, on peut raisonnablement douter de la basquité des deux toponymes navarrais précités. 
31. Lea/Lena: un village de la province de Lugo a pour nom Lea, la forme ancienne étant probablement *Lena. Le nom du fleuve biscaïen qui a son embouchure dans la ville de Lekeitio s'appelle Lea. La chute du $n$ intervocalique étant, comme en galicien, constante en basque, on peut supposer ici aussi que la forme ancienne de cet hydronyme biscaien était ${ }^{*}$ Lena. Un fait viendrait confirmer cette hypothèse : un village asturien a pour nom Lena (consejo ou conseil municipal de Pola de Lena, sud des Asturies). On considère en règle générale qu'il s'agit du vocable pré-romain *lena, "dalle de pierre" présent dans les Alpes et les Pyrénées ${ }^{46}$. En asturien, contrairement à ce qui se produit en galicien, en basque et en gascon, les $n$ intervocaliques ne s'effacent pas. Au XVI ${ }^{\circ}$ siècle, la véritable forme asturienne était Llena car dans cette région l'asturien palatalise systématiquement les $l$-à l'initiale ${ }^{47}$, le nom ayant été par la suite espagnolisé : *lena $>$ Llena $>$ Lena (forme espagnole).

32. Lorbé/Lurbe: le petit village de Lorbé (oxyton), situé à côté de la ville de La Corogne, se trouve en bord de mer dans une région où la côte, surnommée "la Côte de la Mort" ( $A$ Costa da Morte), est très découpée et regorge de falaises. Les formes anciennes de ce nom ne semblent pas, sauf erreur, être connues. Le nom ressemble à celui porté par le village béarnais de Lurbe (Lurbe-Saint-Christau), autrefois Lurbe, 1385, qui s'explique par le basque lur, "terre" et -be, "sous", c'est-à-dire sans doute, d'après Michel Grosclaude, "terre basse". Seules les formes anciennes de ce nom galicien nous permettraient probablement de savoir de quoi il s'agit exactement. Quoi qu'il en soit, ce nom n'a jamais été expliqué.

33. Mahía/La Mahia/Amaya/Amaia/A Mahía : ce nom désigne actuellement une vallée, une paroisse, une montagne, un archiprêtre et une juridiction de $\mathrm{La}$ Corogne. La Mahía est une forme espagnolisée (confusion avec l'article la), A Mahia étant une forme galicienne (confusion avec l'article galicien $a$ ), issues toutes les deux d'une forme plus ancienne Amaia. Dans l'Antiquité, dans le pays de la tribu galaïque des $A_{\text {maaeci }}{ }^{4}$, le territoire appelé Amaya (époque romaine) s'étendait en direction du sud le long de l'actuelle rivière appelée Sar et vers le nord jusqu'au fleuve appelé Tambre. A l'extrémité nord-est de ce territoire, où se trouve aujourd'hui le village d'A Mahia ou Mahia, il y avait autrefois une petite montagne qui se prolongeait vers le nord du côté où la pente était la plus douce. C'est à cet endroit que fut construite la ville de Saint-Jacques-de-Compostelle. Il faudrait peut-être rapprocher ce toponyme des nombreux microtoponymes Amaia qu'on rencontre en Navarre (Leache, Bertizarana et vallée de Linzoáin) et probablement aussi du nom du village appelé Maya (vallée du Baztan, autrefois, Amaia, 1208, Amaya, Amayer, Amayr, 1192-1208, Maia, 1270, Maya, 1234, en basque Amaiur, autrefois amayur, 1208) ainsi que, sans doute, des nombreux toponymes Amaya qui se rencontrent en Espagne, dans les provinces de Burgos où on trouve la localité historique et la montagne (1362 m.) appelées respectivement Amaya et Peña de Amaya qui servaient dans l'Antiquité de frontière méridionale au pays des Cantabres -, Avila, Guadalajara, voire même peut-être, mais ce n'est pas sûr, des toponymes Maya qu'on rencontre dans les provinces de Salamanque et de Grenade. On aurait eu affaire à un mauvais découpage : La Amaya > La Maya > Maya, le sens étant sans doute "confins, limites". En ce qui concerne le toponyme galicien, en théorie la chose ne serait pas totalement invraisemblable car le village appelé A Mahía ou La Mahía, certains auteurs galiciens écrivent Amaia, est situé à l'extrémité nord du territoire ou vallée auquel il son donne son nom. Rappelons également que le village navarrais de Maya ou 
Amaiur est le dernier village de la vallée de Baztan avant le col d'Otxondo (570 m.), les villages de Zugarramurdi et d'Urdax, situés après ce col, ne se trouvant pas à proprement parler dans la vallée. En ce qui concerne l'Amava cantabre, Schulten, qui cite Holder, pense qu'il s'agit d'un nom celtique.

34. Meano : le village navarrais appelé Meano (vallée d'Aguilar, bailliage d'Estella) se retrouve, comme le souligne J. Gifford, dans la province de Pontevedra sous la forme Meaño (avec - $\tilde{n}$-intervocalique).

35. Oka/Oca: le fleuve traversant la ville biscaïenne de Gernika (forme basque et officielle) s'appelle Oka. Le nom se retrouve en Galice. II s'agit du nom de trois villages de La Corogne : Oca, Oca de Abaixo et Oca de Enriba. Pour les toponymes $O c a$, il faut se reporter à l'article d'Alfonso Irigoyen ${ }^{49}$.

36. Oróns/Oronz : le nom du village navarrais appelé Oronz (vallée de Salazar, bailliage de Sangüesa), en basque Orontze, n'est toujours pas expliqué. Un village de la province de La Corogne s'appelle Oróns.

37. Sada: en Navarre, on trouve le village de Sada, 1112, en basque Zare, 1127 (bailliage de Sangüesa). P. Salaberri ${ }^{50}$ a essayé de comparer, en vain comme il reconnaît lui-même, la forme basque Zare avec le toponyme labourdin Sara, sare 1289 (en français Sare). La forme Zare n'est rien d'autre qu'un avatar basquisé et populaire du nom Sada, l'alternance $-d$ - > $r$ - entre voyelles étant extrêmement courante en phonétique basque comme le rappelle Henri Gavel. Ce qu'il faut expliquer, c'est Sada. Il s'agit, comme le rappelle J. Gifford, du même nom que celui de la ville galicienne de Sada située à côté de La Corogne. L'explication du nom donnée par ce dernier est tout à fait satisfaisante : ce toponyme bascogalicien est issu du latin satus, plu. sata, "terres ensemencées" avec sonorisation normale en galicien du - $t$ - intervocalique. En Navarre, la sonorisation, ici d'origine obligatoirement romane, a également eu lieu comme dans le toponyme également navarrais et voisin Artieda, arteda, 1178 (vallée d'Urraúl, Sangüesa).

38. Senari: une inscription funéraire aquitanique découverte près de Luchon (Cazaril-Laspène, Haute-Garonne) porte l'épitaphe suivante : Hotarri. Orcotarris. f (ilio)/Senarri [datif]. Eloni. filiae/Bontar. Hotarris. f(ilius). ex. testamento. Comme le rappellent Julio Caro Baroja, Luis Michelena et Joaquín Gorrochategui, on ne sait pas exactement s'il faut lire Sentarri (d'après Ricci) ou Senarri (d'après Sacaze). En Galice, un hameau de la paroisse de Santiago de Entrambasaguas (municipalité de Guntín, Lugo) a pour nom Sear. L'endroit est dit en 897 Scti. iacobi de Senari quae dicitur de intra ambas aguas. A notre connaissance, ce fait n'est rapporté par aucun auteur. L'amuïssement, normal en galicien, du - $n$ - intervocalique explique la forme moderne Sear. Dans dans la province de Pontevedra, un hameau porte le même nom. En outre, l'Itinéraire d'Antonin cite chez les Astures augustani (actuelle province de Léon) un lieu appelé Uttaris (probablement le village appelé Ruitelán, Léon) qui ressemble au nom aquitanique Hotarris (génitif). Dans sa récente thèse de doctorat ayant pour objet l'anthroponymie médiévale galicienne du VIII ${ }^{e}$ au XIII ${ }^{e}$ siècle, Ana Isabel Boullón Agrelo cite les noms de personne galiciens Oihenia et Hahanxi, les deux étant féminins, qu'elle compare au vocable basque oi(h)an, "forêt" et à l'anthroponyme féminin aquitain Hahanni figurant sur une inscription retrouvée dans la ville de Saint-Bertrand-de-Comminges (Haute-Garonne). 
39. Tamariz/Tamariz/Tamaris/Támara/Tambre : Julio Caro Baroja si cite le toponyme Tumariz qui désigne, précise-t-il, une montagne navarraise. Il s'agit également, dit Julio Altadill, d'une corraliza ou basse-cour située sur la route qui va d'Alfaro à l'Ebre. On retrouve le toponyme dans le nom de lieu galicien Tamariz cité par D. Ramón Menéndez Pidal. Il s'agit également de la forme ancienne du nom du fleuve galicien connu aujourd'hui sous le nom de Tambre (Lü Corogne) : Tamaris flumen (époque romaine), uillam de anna in Pistomarcos in ripa Tamaris, $\mathrm{X}^{\mathrm{e}}$ siècle > Tamare, $934>$ Tambre. D'après notre auteur, il représente le peuple, non celtique selon Meyer-Lübke, des Tamarici ou Tamarak cité par Mela et Pline et qui habitait au bord du fleuve galicien Tamaris, également connu par les auteurs greco-latins sous le nom de Tamara, nom qui est encore de nos jours porté par une rivière de La Corogne : río Támara (proparoxyton). Il s'agit en outre d'un prénom encore utilisé de nos jours dans le Caucase, une reine de Géorgie (1184-1212) l'ayant autrefois porté. Ptolémée dit qu'il y avait dans le pays des Cantabres (c'est-à-dire grosso modo l'actuelle communauté autonome de Cantabrie, cette région étant également surnommée La Montaña) une ville appelée Tamarica, capitale probable, d'après Julio Caro Baroja $\mathrm{a}^{52}$, de la nation ou lignée cantabre des Tamarici. Certains auteurs lisent Camarica au lieu de Tamarica (cf. suffixe -ica/-ika, §30).

40. Treviño : en Pays Basque, on trouve le comté de Treviño. Bien qu'enclavé dans la province d'Alava, il dépend d'un point de vue administratif de la province espagnole de Burgos. Le nom est issu, dit Julio Caro Baroja, du latin. Il est également porté par un village de La Corogne.

41. Tena/Tea : bien qu'elle ne soit pas située en Pays Basque, il faut citer la rivière aragonaise appelée de nos jours Gállego (proparoxyton) qui était située dans l'antiquité dans le territoire des Ilergetes, voisins des Vascones. Elle devait servir vraisemblablement, d'après Julio Caro Baroja, de frontière à ces deux peuples. Au moyen âge, elle est appelée Gallico, Gallecum, Gallegum, Gallicus, $\mathrm{XI}^{\mathrm{c}}$ siècle. Cette rivière aragonaise divise en deux parties une vallée connue sous le nom de Tena (valle de Tena), dont le nom est déjà documenté en 1076: usque ad montes gaelicos cum tota Tena ${ }^{33}$. Ce nom se retrouve en Galice, dans la province de Pontevedra. Il désigne une vallée et une rivière : rio Tea et valle de Tea, autrefois, Tena, ripa Tenae, XII ${ }^{\star}$ siècle. La chute du - $n$-intervocalique est normale en galicien.

42. Ulia/Uli/Uría : les toponymes navarrais Uli (village d'Uli-Alto, Uli en 1052 , vallée d'Arce, bailliage de Sangüesa) et Uli (vallée de Lónguida, Sangüesa) sont considérés comme étant d'origine inconnue. Au XVI siècle, les bascophones appelaient ces endroits Uli (aujourd'hui Ulibeiti d'après Euskaltzaindia) et Uliberri, "nouvel Uli". Mais le nom n'aurait, semble-t-il, aucun rapport avec le nom également navarrais Ulibarri, "ville neuve", Uriuarri la mayor, 1277 (vallée de Lana, bailliage d'Estella) comme l'indique Julio Caro Baroja qui rappelle que le nom de la vallée d'Arce "en la parte occidental y meridional de navarra podría equipararse a "uli" o "uri" en compuestos. No aqui" ${ }^{\prime \prime}$. Le nom étant considéré comme énigmatique, aucun auteur n'émet d'hypothèse. Le nom navarrais Uli paraît correspondre à celui du fleuve galicien appelé aujourd'hui Ulla, autrefois fluuii Ulia, 883 ; Ulia, 934 ; la palatalisation ultérieure de la latérale étant normale en galicien. Le nom ancien de ce fleuve galicien est identique, comme l'avait déjà noté Humboldt ${ }^{55}$, à celui que porte encore de nos jours la montagne 
guipuscoane appelée Ulia (227 m.), située entre Saint-Sébastien et Le Passage de Saint-Jean et dont la signification exacte n'est pas connue. Une des chaines de montagnes qui séparaient dans l'Antiquité les Galaïques des Astures a de nos jours pour nom Sierra de Lría. Peut-être faut-il également rapprocher cet oronyme asturien, dont la forme ancienne nous est inconnue mais qui semble cependant être très ancien, de celui qu'on trouve en Guipuscoa ainsi que de l'hydronyme galicien. Strabon (Géographie, III, 2, 1) mentionne plusieurs villes du sud de l'Espagne (actuelle Andalousie), dont une située sur une montagne et appelée Ulia : "Après ces villes viennent [les villes d'] Italica et llipa, situées sur le Bétis [Guadalquivir], Astigis, à quelque distance du fleuve, Carmo, Obulco; puis, dans la région où furent vaincus les fils de Pompée, Munda, Atégua, Urso, Tuccis, Ulia et Aegua, toutes peu éloignées de Corduba [Cordoue]". Humboldt avait déjà souligné l'équivalence Ulia galicien = Ulia guipuscoan (où le groupe vocalique $-i a-$ ne forme pas une diphtongue et dont le nom est "graphié" Ulía en espagnol) = Ulia ville de la Bétique (époque romaine).

43. Uriz/Oriz : les noms des villages navarrais de Úriz, en basque Uritz (vallée d'Arce) et de Óriz, en basque Oritz (vallée d'Elorz, bailliage de Sangüesa) se retrouvent dans ceux des villages galiciens appelés Uriz et Oriz (province de Lugo). La seule différence qui existe entre eux est la suivante : les galiciens sont oxytons alors que les formes espagnoles des toponymes navarrais sont accentuées sur la pénultième.

44. Urrós: dans la municipalité galicienne d'Allariz (Orense), on trouve un hameau appelé Urrós (oxyton). Ce nom est apparemment identique, comme le signale $\mathbf{J}$. Gifford, à ceux portés par les villages navarrais d'Urrotz (vallée de Malerreka, Pampelune) et d'Urroz (bailliage de Sangüesa). G. Rohlfs cite la localité aragonaise appelée Urriés ${ }^{56}$. Si le toponyme Urriés est issu, comme le pense cet auteur, de l'anthroponyme non attesté *Urrus, ce qui est cependant peu probable dans le cas du toponyme aragonais, alors il devrait en être de même pour le nom galicien Urrós, avec cependant ici une déclinaison en -o, -oris (génitif) au lieu du suffixe aquitanique : *Urrus $>$ *urronis $>$ *urrones (avec évolution romane et normale du $-i$ - dans -onis qui passe à -ones) $>*$ urroes (chute normale du $n$ intervocalique) $>{ }^{*}$ urrois $>$ urrós. Quoi qu'il en soit, ce nom galicien reste inexpliqué.

45. Ustariz: dans la municipalité d'Otero de Rei (Lugo), une petite montagne est connue sous le nom de Monte de Ustariz. Cet oronyme galicien est probablement un anthroponyme d'origine germanique, sans doute Ost-/Austericus (cf. M.Th. Morlet) $>\left(\right.$ villa) ${ }^{*}$ Osterici $>$ Osteriz/Ostariz/Ustariz. On le retrouve vraisemblablement dans le toponyme navarrais Osteritz, autrefois Ostariz, 1280 (vallée d'Esteribar, Sangüesa), étant donné que plusieurs villages navarrais, portent un nom germanique comme, par exemple, celui appelé Oderitz (vallée de Larraun, bailliage de Pampelune) dont l'origine germanique est établie : Odericus attesté en 861 (cf. Marie-Thérèse Morlet), d'où (villa) ${ }^{*}$ Oderici $>$ Oderiz, en basque Oderitz. En ce qui concerne le toponyme labourdin Ustaritz, il faut se reporter à l'ouvrage de Jean-Baptiste Orpustan.

46. Viscaya: un hameau de la paroisse de San Pedro de Láncara (Lugo) s'appelle Viscaya, sans qu'on en connaisse la raison exacte. Une région située dans la valiée navarraise d'Aibar est égaiement appeiée Viscaya, le nom étant orthographié le plus souvent en basque Bizkaia. 
47. - car : on n'a pas réussi à expliquer les toponymes navarrais Añézcar (Ansoáin, bailliage de Pampelune), Ucar (Valdizarbe, bailliage de Pampelune) et Osácar (vallée de Juslapeña, bailliage de Pampelune), l'élément final-car ne pouvant être éclairci. On a essayé toutefois de rapprocher le premier du vocable souletin ainazkar, añazkar; "bruyère", les deux autres restant complètement énigmatiques. Dans la province de La Corogne, on trouve de nos jours le patronyme galicien Igléscar et dans la province d'Orense un village appelé Anicar, l'élément final étant inexpliqué. En Espagne, on trouve les toponymes Iscar (Valladolid, Castillela-Vieille) et Izcar (Cordoue, Andalousie) où l'élément -car apparaît également.

Cet article ne constitue qu'un simple survol d'un sujet qui demande à être approfondi car la toponymie présumée d'origine pré-indo-européennne est très abondante en Galice et malheureusement trop souvent délaissée. Le fait que plusieurs cartulaires galiciens, représentant des milliers d'archives médiévales, n'aient toujours pas été, on l'a dit, dépouillés, étudiés et édités constitue malheureusement encore un frein en ce qui concerne la poursuite de ces recherches toponymiques.

D'autre part, si on écarte les similitudes toponymiques dans lesquelles nous avons manifestement affaire à des anthroponymes d'origine latino-romane, voire germanique, similitudes toponymiques galaïco-basques, parfois hypothétiques, souvent indéniables, qui demanderaient cependant à être expliquées, il reste plusieurs similitudes qui posent problème.

En effet, il reste à expliquer le pourquoi des doublets toponymiques galaïcoguipuscoano-biscaïens Ezaro/Izaro, Lea/Lea (et le Lena asturien), Oka/Oca, Ulia/Ulia (et peut-être les deux Uli navarrais), Deva/Deba, Ego/E(g)o, Igueldo/Gueldo, pour ne citer que les plus curieux d'entre eux et, surtout, à expliquer pourquoi ils ne peuvent pas manifestement s'expliquer par le basque.

Cela nous renvoie à un vieux débat qui n'a jamais été résolu de façon définitive et qui concerne la basquité originelle des Caristes et des Vardules ou, si on préfère, la basquité première des Biscaïens et des Guipuscoans de l'Antiquité. Il s'agit d'une question très ardue comme le reconnaissent, entre autres, Joaquín Gorrochategui et Luis Michelena.

A. Schulten ${ }^{57}$ fut l'un des premiers à défendre l'idée selon laquelle les Caristes et les Vardules, et à plus forte raison, les Autrigones (les habitants grosso modo des actuelles Encartaciones biscaïennes et de la partie nord-est de la province de Burgos) et les Berones (c'est-à-dire grosso modo les actuels habitants de la Rioja) n'étaient peut-être pas des proto-Basques mais, au contraire, des peuples d'origine indo-européenne ou, tout au moins, des peuples parlant une langue indo-européenne. Mais ce sont surtout, reconnaissait Luis Michelena, les travaux du savant espagnol D. Manuel Gómez-Moreno ${ }^{58}$, à l'origine du déchiffrage de l'alphabet ibérique, qui vinrent appuyer de manière convaincante cette thèse.

Pour contourner la difficulté posée par les travaux de ce dernier et dont la valeur était incontestable, ceux de Schulten étant plus discutables, Luis Michelena et par la suite Joaquin Gorrochategui firent appel à la sociolinguistique. Autrement dit, les Caristes et les Vardules - les Vascones posant également un autre problème que nous n'aborderons pas ici, l'origine celtique des Autrigones et des Berones étant généralement admise - utilisaient une anthroponymie et une toponymie indo-européennes tout en parlant basque ou plutôt proto- 
basque. Luis Michelena, qui manifestait une certaine réticence à l'égard de l'idée selon laquelle les Caristes et les Vardules n'étaient pas des proto-bascophones, reconnaissait cependant que "aunque nos esforcemos de atenuar por todos los medios la fuerza de estos testimonios [indo-européens en Pays Basque], tendremos que acceptar que su coincidencia dificilmente puede explicarse sin admitir que gentes de habla vasca conocían y usaban también, en mayor o menor número, algún dialecto indoeuropeo" "39. On le voit, Luis Michelena admettait que les Caristes et les Vardules devaient nécessairement parler une langue autre que le proto-basque, mais il préférait cependant penser, contrairement à D. GómezMoreno, qu'il s'agissait quand même de proto-Basques.

A cela vient s'ajouter encore une autre question : celle de l'origine des dialectes basques, notamment ceux du Pays Basque méridional. Tous les spécialistes admettent qu'en basque les divergences dialectales s'estompent au fur et à mesure que l'on remonte dans le temps jusqu'aux premiers textes basques connus, ce qui à terme signifie nécessairement, comme le rappelle Luis Michelena ${ }^{60}$, qu'il a dû exister autrefois une langue commune.

Là où la question prend une tournure des plus complexes est qu'on ne s'explique pas alors "la remarquable similitude - souligne Beñat Oyharçabal - entre les aires couvertes par le navarrais et biscaïen en Guipuscoa, et les territoires occupés il y a deux mille ans les Vascones et les Caristii dans leur voisinage avec les Varduli" ${ }^{61}$. Autrement dit, en se basant sur les données fournies par le géographe Ptolémée, les démarcations territoriales qui étaient dans l'Antiquité celles des peuples ou tribus appelés respectivement Caristes, Vardules et Vascones correspondent exactement aux aires dialectales modernes du biscaïen, du guipuscoan et du haut-navarrais septentrional, tout au moins dans leur partie septentrionale là où la langue s'est conservée jusqu'à nos jours. Cela sous-entend qu'au début de notre ère les principaux dialectes méridionaux existaient déjà, ce qui semble être manifestement en contradiction avec le fait, admis par tous les spécialistes, qu'en basque les divergences dialectales s'atténuent au fur et à mesure qu'on remonte dans le temps.

Pour contourner cette difficulté, Julio Caro Baroja proposa une hypothèse allant dans le sens de celle émise par D. Gómez-Moreno. D'après lui, il n'était pas invraisemblable de supposer que les Vardules et les Caristes constituaient des fractions issues de la nation des Cantabres qui, ayant acquis une certaine importance au fur et à mesure passait le temps, seraient devenues "autonomes", c'està-dire qu'on aurait eu affaire à "una desmenbración de la estirpe cántabra, parecida a otras que se observaron en sociedades regidas por el sistema de linajes en que, pasando un número determinado de generaciones, las ramas van tomando nombres propios" étant donné, ajoute-t-il, que les inscriptions de l'époque romaine "nos reflejan que en este orden várdulos y caristios eran parientes, afines, de los cántabros y astures" et que par conséquent cela "nos obliga a plantearnos de nuevo el problema del que pudiéramos llamar cantabrismo de los pueblos del Noroeste litoral, que una crítica rigurosa parecía haber reducido a cero" ${ }^{62}$.

En résumant un sujet qui est complexe, les tribus, présumées alors cantabriques, des Caristes (Biscaïens) et des Vardules (Guipuscoans) et parlant une langue indéterminée, peut-être indo-européenne, mais nécessairement différente du proto-basque, auraient, à une date et pour des raisons qui nous échappent, été basquisées ou plutôt "vasconnisées". Il faut reconnaître que cette hypothèse pré- 
sente quelques avantages : $1^{\circ}$. elle expliquerait pourquoi les anciens territoires des Caristes et des Vardules correspondent exactement aux actuelles divisions dialectales méridionales; autrement dit, ces deux peuples de l'antiquité se seraient mis à parler proto-basque tout en conservant dans leur manière de le parler certains traits de leur langue primitive et jusqu'à présent indéterminée, ce qui expliquerait la singularité du parler guipuscoan et surtout celle du dialecte biscaïen; $2^{\circ}$. elle expliquerait pourquoi Jules César, relatant la conquête de l'Aquitaine menée par son lieutenant Crassus en 56 av. J.-C., parle "des cinquante mille hommes fournis par l'Aquitaine et le pays des Cantabres" ${ }^{63}$, ces derniers étant accourus au secours des premiers, quoique certains y aient vu une erreur - hypothétique cependant - de César qui aurait confondu les Caristes et les Vardules avec les Cantabres "authentiques"; $3^{\circ}$. elle expliquerait, enfin, pourquoi on retrouve en Galice certains toponymes, hydronymes et oronymes biscaiens et guipuscoans apparemment inexplicables par le basque.

Néanmoins, cette hypothèse sur le cantabrisme présumé des peuples du nordouest de la péninsule n'explique pas pourquoi les Caristes, les Vardules et les Autrigones, s'ils appartenaient véritablement à la nation des Cantabres, ne prirent pas part, pas plus que les Vascones d'ailleurs, à la guerre que les Galiciens, les Asturiens et les Cantabres menèrent contre Rome.

Une guerre totale qui dura dix ans $\mathrm{s}^{64}(29-19$ av. J.-C.) et qui provoqua la venue sur place de l'empereur Auguste en personne, ce dernier allant même jusqu'à faire une dépression. II faudrait aussi supposer que les Autrigones n'auraient pas été affectés par cette présumée "vasconnisation" dont nous avons fait mention puisque, semble-t-il, il n'existe pas d'éléments tendant à montrer un emploi autrefois de la langue basque dans ce qui constituait leur territoire, pays qui englobait, entre autres, les actuelles Encartaciones biscaiennes.

Une autre possibilité effleurée, entre autres, par Julio Caro Baroja, est de supposer que les Caristes et les Vardules n'appartenaient plus à la nation des Cantabres tout en étant néanmoins d'origine "cantabroïde" (et non pas "vascoïde") ou encore qu'ils n'étaient ni d'origine "cantabroïde" ni "vascoïde" " origine restant indéterminée.

Cela étant, on ne sait toujours pas quelle était l'origine exacte des Cantabres et des Astures, pas plus que celle des Galiciens, excepté qu'il est certain qu'il y avait parmi ces peuples, comme au demeurant chez les Caristes et les Vardules, des populations d'origine celtique. On pourrait également admettre que les Caristes et les Vardules étaient d'origine vascoïde ou, si on préfère, qu'ils parlaient proto-basque, mais cela implique nécessairement, pour les raisons évoquées plus haut, que les dialectes appelés de nos jours biscaïen et guipuscoan existaient déjà il y a deux mille ans, ce qui s'avère également problématique.

L'affaire, on l'aura compris, est complexe car d'un autre côté plusieurs toponymes galiciens ne semblent pas explicables par une langue celtique.

\section{Hector IGLESIAS}

Doctorant en Études Basques 


\section{Bibliographie}

ALBERTOS FIRMAT, M². L., La Onomástica personal primitiva de Hispania : Tarraconense y Bética, Université de Salamanque, 1966.

ALTADILL, J., Geografía General del Reino de Navarra, 2 vol., 1914.

AGUD, M. \& TOVAR, A., " Diccionario etimológico vasco ", Anejos del Anuario del Seminario de Filologia Vasca "Julio de Urquijo", XIII, XIX, XXIV, XXVI, XXX, XXXVII, T. I, II, III, IV, V, VI, VII, Saint-Sébastien, 1989-1993.

ASJU = Anejos del Anuario del Seminario de Filologia Vasca "Julio de Urquijo".

AZKUE, R.-M., Diccionario Vasco-español-francés, Bilbao, rééd. 1984.

BELASKO, M., Diccionario elimológico de los nombres de los pueblos, villas y ciudades de Navarra, Éd. Pamiela, Pampelune, 1996

BOULLÓN agrelo, A. I., Contribución o esiudio da antroponimia medieval galega (séculos VIII-XIII), Thèse doctorale inédite, Université de Saint-Jacques-de-Compostelle, 1994, exemplaire microfilmé.

BRSVAP = Boletin de la Real Sociedad Vascongada de los Amigos del Pais.

BSSLAP = Bulletin de la Sociéti des Sciences, Letres' \& Arts de Puu.

CABEZA QULLES, F., Topónimos de Galicia : a süa orix" e o seu significado, Éd. Montes c lontes, Xerais, 1992.

CARO BAROJA, J., Materiales para una historia de la lengua vasca en su rilación con la latina. Estudios Vascos, XIX, Université de Salamanque, 1945, rééd. Éd. Txertoa, Saint-Sébastien, 1990.

- Sobre el mundo ibérico-pirenaico : datos, nuevas ideas sobre el lberismo, Fistudios Vascos, XVIII, Éd.. Txertoa, Saint-Sébastien, 1988.

- Los pueblos del norte de la Peninsula lberica, 3c éd., Txertoa, Saint-Sébastien, 1977.

_- Los Vascones y sus vecinos. Estudios Vascos, T. XIII, Txertoa, Saint-Sébastien, 1985.

, "Los estudios geografico-históricos sobre el pais vasco y la dialectologia", in Sobre la religión antigua y el calendario del pueblo vasco, Estudios Vascos, T. I., Éd.. Txertoa, Saint-Sébastien, 1984.

_ Sobre la lengua vasca. Estudios Vascos, T. IX, Ed.. Txertoa, $2^{e}$ éd, Saint-Sébastien, 1988

- Etnografia Histórica de Navarra. Caja de Ahorros de Navarra, Pampelune, 1972.

COROMINES, J. \& PASCUAL, J. A., Diccionario critico etimológico castellano e hispánico, 5 vol, Gredos, 1980 .

COROMINES, J., "Elementos prelatinos en las lenguas romances hispánicas", Actas del I Coloquio sobre lenguas y culturas prerromanas de la Peninsula lbérica, Salamanca, 1974. 1972, pp. $299=319$.

"De toponimia vasca y vasco-románica en los Bajos-Pirineos", $F L V, n^{\circ} 12$, Pampelune Heidelberg, 193-308.

, "Du nouveau sur la toponymie occitane", Beiträge zur Namensforschung. 8. 1973,

DAUZAT, A. \& ROSTAING, Ch., Diclionnaire étymologique des noms de lieux en France, Paris, nouvelle édition 1989 .

DIEZ MELCÓN, G., Apellidos castellano-leaneses (siglos IX-XIII, ambos inclusive), Université de Grenade, 1957.

EUSKALTZAINDIA, Onomasticon Vasconiae 4. Actas de las I jomadas de onomástica, toponimia, Vitoria-Gasteiz, abril 1986/1 onomastika jardunaldien agiriak. Toponimia, Gasteiz, $1986 k o$ apirila, Ed.. Euskaltzaindia, 1991. 
, Nafarroako herri izendegia/Nomenclátor euskérico de Navarra, Pampelune, 1990.

FERNÁNDEZ REI, F, Dialectoloxia da lingua galega, Ed. Xerais, 1991

FLV $=$ Fontes Linguae Vasconum. Institución Príncipe de Viana. Pampelune.

GARCIA ARIAS, X. L.I, Pueblos asturianos : el porqué de sus nombres. Ėd.. Ayalga, Collección Popular Asturiana, Gijón, 1977.

GAVEL, H., "Éléments de phonétique basque", RIEB, 1921, XII, an 15.

," "Remarques sur les substrats iberiques, réels ou supposés dans la phonétique du gascon et de l'espagnol", Revue de Linguistique Romane, T. XII, 1936, n 45-46.

"Du nom de Bayonne et de quelques autres : noms de lieux aquitains ou espagnols", BSSLAERB, $\mathrm{n}^{\circ} 7,1931$.

GGRG = Geografia General del Reino de Galicia, 13 vol., sous la direction de F. Carreras y Candi, Ediciones Gallegas, Editorial La Gran Enciclopedia Vasca, La Corogne-Bilbao, 1980

GIFFORD, J., "Topónimos gallegos y topónimos navarros", BRSVAP, 1955, XI, pp. 227-231.

GÓMEZ-MORENO, M., "Sobre los iberos y su lengua", Homenaje ofrecido a Menéndez Pidal, III, 1925, pp. 225-240.

GORROCHATEGUI CHURRUCA, J, Estudio sobre' la onomástica indigena de Aquitania, Éd.. Université du Pays Basque (UPV-EHU), Bilbao, 1984.

GOYHENECHE, 1.. Onomastique du Nord du Pays Busque au Moyen Age. XI-XVe siècles, thèse de doctorat dactylographiée, Bordeaux, 1966

GROSClAUDi:, M., Dictionnaire toponymique des communes du Béarn, Pau, 1991.

GULÍAS LAMAS, X. A., Beariz de Montes: Aspecto antropolóxico, histórico e cultural dun pobo, Éd.. Concello de Beariz, Orense, 1992.

HOLDER, A., Altceltischer Sprachschutz, Leipzig, 1907-27.

HUMBOLDT, W. von, Prüfung. Prüfung der Untersuchungen über die Urbewohner Hispaniens vermittelst der Vaskischen Sprache, 1921, Imp. F. Dümmler, Berlin, 192 pages. Traduction espagnole de $\mathrm{T}$. Aranzadi: "Examen de las investigaciones sobre los aborigenes de España mediante la lengua vasca, traducción directa del alemán (2e edición) por Telesforo de Aranzadi", RIEV, 1934, pp. 475-520 et RIEV, 1935, pp. 44-92 ; pp. 499-552.

IGLESIAS, H., "Le toponyme Biarritz", FLV, n 78, 1998, pp. 281-288.

IRIGARAY, A. \& MICHELENA, L., "Nombres vascos de persona", BRSVAP, I955, XI, pp. 405-425.

IRIGOIEN, A., Sobre toponimia del pais vasco norpirenaico, Université de Deusto, 1990.

" "Sobre los topónimos Oca y su entorno". Symbolae Ludovico Mitxelena septuagenario oblatae. Universite du Pays Basque (UPV-EHU), Instituto de (iencias de la Antigüedad, Vitoria, 1985, pp. $1007-1017$ cae IV, Bilbao, 1992, pp. 213-224

"Leku-izenen euskarazko formak Nafarroan", De re philologica linguae uasconicae, Bilbao, 1987, pp. 263-264.

JAUPART, F., Le nom de Bayonne, les lieux, les personnes. T. I et II, Bayonne, 1981.

JORDÁN CÓlERA, J., "De la raiz *IZ- "agua" en vasco", FLY, nº 78, 1998, pp. 267-279.

JUNGEMANN, F., La teoria del sustrato y los dialectos hispano-romances y gascones, Madrid, 1955.

LAFON, R., "Noms de lieux d'aspects basque en Andalousie", tiré à part des Actes et Mémoires du $5^{z}$ Congrès International de sciences onomastiques, vol, I, Salamanque, 1958, p. 3-12. 
LECUONA, M., "El euskera en Navarra a fines del siglo XVI", Geografia histórica de la lengua vasca (siglos XVI al XIX), Collección Auñamendi, 1960.

LOUANDRE, Ch., Commentaires de Jules César. Guerre des Gaules, Traduction nouvelle avec le texte, des notes et un index, Éd. E. Fasquelle, Paris, 1931.

LUCHAIRE, A., "Du mot basque IRI et de son emploi dans la composition des noms de lieux de l'Espagne et de l'Aquitaine antique", BSSLAP, 1874-1875, pp. 18-27.

MADOZ, P., Diccionario geográfico-estadístico-histórico de España y sus posesiones de Ultramar, 16 vol., Madrid, 1845-1850.

MARTÍneZ BARBEITO, C. Galicia. Éd.. Destino, Barcelone. 3= éd., 1971.

MELA, P., Eurres complètes, avec lia traduction en français, publiées sous la direction de M. Nisard de l'Académie Française, Inspecteur Général de l'Enseignement Supérieur, Éd. Firmin-Didot, Paris, 1875.

MENÉNDEZ PIDAL, R., Origenes del español, 7e éd., Espasa Calpe, Madrid, 1972.

Toponimia Prerrománica Hispánica, Gredos, Madrid, 1968.

MICHELENA, L., Apellidos Vascos. $3^{\circ}$ éd., Txertoa, Saint-Sébastien, 1973.

, Textos arcaicos vascos, Éd. Minotauro, Madrid, 1964, ASJU, Saint-Sébastien, 1990.

"Sobre la historia de la lengua vasca", ASJU, XVI, 1982, pp. 143-156.

"Lengua comun y dialectos vascos", ASJU, XV, 1981, pp. 29 I-313.

Sobre el pasado de la lengua vasca. Coll. Auñamendi, Saint-Sébastien, 1964. 1977.

MORALEJO I.ASSO, A., Toponimia gallega y leonesa, Éd. Pico sacro, Saint-Jacques de Compostelle,

, "Observaciones sobre el estudio de la toponimia gallega", Cuademos de Estudios Gallegos, I, 1944, pp. 59-72.

"Ojeada a los topónimos hispánicos y especialmente a los gallegos de origen prelatino de J. Corominas", Verba 5. Anuario galego de filoloxia, pp. 13-24, Université de Saint-Jacques-deCompostelle, 1978.

MORLET, M.-Th., Les noms de personne sur le territoire de l'ancionne Gaule du V'" au XI" siècle. ILes noms issus du germanique continental et les créations gallo-germaniques. II.- Les noms latins ou transmis par le latin, Paris, CNRS, 1972.

OLANO SILVA, V., "Toponimia gallega", RDTP, I., 1945, pp. 653-666; V., 1949, pp. 626-662; X., 1954, pp. 190-226.

ORPUSTAN, J.-B., Toponymie basque, Presses Universitaires de Bordeaux, 1990.

OYHARÇABAL, B., "Variété dialectale et unification littéraire", in Euskal Herriak/Pays Basques, T. II, Les cahiers de l’IFOREP n ${ }^{\circ}$ 57, Paris, 1989

PIEL, J.-M., Os nomes germánicos na toponimia portugesa, I. Adaes-Novegildo. Lisbonne, 1937. II. Oldroes-Zendo. Lisbonne, 1945

23, 1948, pp. 143-202.

"Nomes de posssessores latino-cristãos na toponimia asturo-galego-portuguesa", Biblos,

PLINE L'ANCIEN, Histoire naturelle de Pline, avec la traduction en français, par M. Émile Littré, Paris, $1850-1851,2$ vol.

PTOLÉMÉE, C., Klaudiou Ptolemaiou Geógraphikê uphêgêsis Claudii Ptolemaei Geographia. E codicibus recognovit, prolegomenis, annotatione, indicibus, tabulis instruxit Carolus Müllerus. Voluminis primi pars prima. Parisiis, A. Firmin-Didot, 1883, T. I, 1901, T. II.

RAYMOND, P., Dictionnaire toporraphique des Basses-Pyrénées, Paris, 1863. 
RAVIER, X., "Le suffixe toponymique pyrénéen "-un". Le problème de ses relations avec d'autres suffixes à caractéristique nasale de I'Ibéro-Aquitain", Via Domitia, XIL, Fasc. 5, 1963, pp. 57-86.

RDTP $=$ Revista de Dialectologia y Tradiciones Populares.

RFE = Revisia de Filologia Española.

RIEB = Revue Internationale des Études Basques.

ROHLFS, G., Le Gascon. Études de philologie pyrénéenne (avec 3 cartes), 2c édition, entièrement refondue, Tübingen-Pau, 1970.

- "Una forma no investigada en la toponimia del sur de Francia y de la España septentrional (el sufijo -és, -iés)", traduit du français par J. M. Enguita, Archivo de Filologia Aragonesa. XL, 1955.

_"Sur une couche préromane dans la toponymie de Gascogne et de l'Espagne du nord", RFE, 26, 1952, pp. 209-256.

ROMÁNI MARTÍNEZ, M., , Colección diplomática do mosteiro cisterciense de Santa Maria de Oseira (Ourense). Consellería de Cultura, Saint-Jacques-de-Compostelle, 1989.

SALABERR1, P., Eslaba aldeko euskararen azterketa topomimiaren bidez. Onomasticon vasconiae 11, Euskaltzaindia, Bilbao, 1994

SUÁREZ, J. C., Toponimia lenense : origen de algunos nombres en tomo al Valle del Huerna. Real Instituto de Estudios Asturianos, Oviedo, 1992.

SAINT-IOUAN, R., Le nom de famille en Béarn et ses origines suivi du Dictionnaire anthroponymique du Beam pour l'an 1385, t. I et II, 1966, réed. Librairie du Palais, 1992.

BSSLAERB = Bulletin de la Société des Sciences. Lettres, Arts \& Etudes Régionales de Bayonne.

SCHULTEN, A., Los cántabros y astures y su guerra con Roma. Madrid, 1943. 1927 , pp. 225-240.

STRABON, Géographie, texte établi et traduit par François Lasserre, Collection des Universités de France, Éd. Les Belles Lettres, Paris, 1966, 2 vol.

URTASUN ANTZANO, E., "Fiteroko hainbat toki izen. Hausnarketa”, FLV n 76, 1997, pp. 497-506. 


\section{Notes}

1. "Elementos prelatinos en las lenguas romances hispánicas", Actas del I Coloquio sobre lenguas y culturas prerromanas de la Península Ibérica, Salamanca, 1974.

2. Toponimia Prerrománica Hispánica, Gredos, Madrid, 1968.

3. "Remarques sur les substrats ibériques, réels ou supposés dans la phonétique du gascon et de l'espagnol", Revue de Linguistique Romane, T. XII, 1936, nª 45-46.

4. La teoria del sustrato y los dialectos hispano-romances y gascones, Madrid, 1955.

5. Sobre la lengua vasca, Estudios Vascos, T. IX, Éd. Txertoa, 2־ éd, Saint-Sébastien, 1988, p. 50.

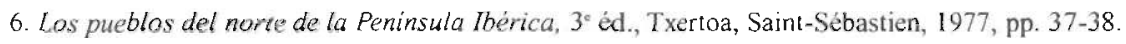

7. CARRE ALDAO, E., GGRG = Geografia General del Reino de Galicia. I 3 vol., sous la direction de F. Carreras y Candi, Ediciones Gallegas, Editorial La Gran Enciclopedia Vasca, La Corogne-Bilbao, 1980, in "Vias romanas y viejos caminos", T. I, chap. X, pp. 265-280; "Demarcaciones históricas", T. I, chap. XI, pp. 281-311.

8. GAVEL, H., "Du nom de Bayonne et de quelques autres". BSSLAERB, n 7, 1931, pp. 38-39.

9. EUSKALTZAINDIA, Onomasticon Vasconiae 4. Actas de las I jornadas de onomástica, toponimia, Vitoria-Gasteiz, abril 1986/l onomastika jardunaldien agiriak. Toponimia, Gasteiz, 1986ko apirila. Ed. Euskaltzaindia, 1991, p. 221.

10. Sobre la lengua vasca, Estudios Vascos, T. IX, Ed.. Txertoa, $2^{*}$ ed, Saint-Sébastien, 1988, p. 51.

11. CABF.7.A QI,ILES, F, Toponimos de Galicia : a súa orixe e o seu significado. Ed.. Montes e fontes, Xerais, 1992, p. 9.

12. CABEZA QUII.ES, F., ur cit., p. 10.

13. "Sur une couche préromane dans la toponymie de Gascogne et de l'Espagne du nord", RFE, 26, 1952, p. 227.

14. Apellidos Vascos, $\$ 18$. p. 170.

15. "Nomes de posssessores latino-cristãos na toponimia asturo-galego-portuguesa", Biblos, 23, 1948 ,

16. Sobre toponimia del pais vasco norpirenaico, Université de Deusto, 1990, pp. 16-17.

17. Apellidos Vascos, \$ 54.

18. LECUONA, M., "El euskera en Navarra a fines del siglo XVI", Geografia hisiórica de la lengua vasca (siglos XVI al XIX). Collección Auñamendi, 1960, p. 134.

19. COROMINES, J. \& PASCUAL, J. A, Diccionario critico etimológico castellano e hispánico, 5 vol, Gredos, 1980, T. I, p. 325.

20. "Sur une couche préromane dans la toponymie de Gascogne et de I'Espagne du nord", RFE, 26, 1952, pp. 229-230.

21. Op. cit., pp. 53-54

22. JAUPART, F., Le nom de Bayonne, les lieux, les personnes. T. I et II, Bayonne, 1981, p. 17.

23. Toponimia Prerrománica Hispánica, Gredos, Madrid, 1968, p. 32.

24. Materiales para una historia de la lengua vasca en su relación con la latina, Estudios Vascos, XIX, Université de Salamanque, 1945, rééd. Éd. Txertoa, Saint-Sébastien, 1990, p. 216.

25. GULIAS LAMAS, X. A., Beariz de Montes : Aspecto antropolóxico, histórico e cultural dun pobo. Ed. Concello de Beariz, Orense, 1992.

26. IGLESIAS, H., "Le toponyme Biarritz", FLV, n 78, 1998, pp. 281-288. 
27. Toponymie basque, $\$ 6$.

28. Op. cit.. $§ 53$.

29. DÍEZ MELCÓN, G., Apellidos castellano-leoneses (siglos $1 X$-XIII, ambos inclusive), Université de Grenade, 1957, p. 123.

30. Textos arcáicos vascos, p. 25.

31. DAUZAT, A. \& ROSTAING, Ch., Dictionnaire étymologique des noms de lieux en France, Éd.. Guénégaud, Paris, nouvelle édition 1989, p. 124.

32. DAUZAT, A. / ROSTAING, Ch., op. cit., p. 125.

33. Toponimia Prerrománica Hispánica, Gredos, Madrid, 1968, p. 119 , n. 29.

34. AGUD, M. \& TOVAR, A., Diccionario etimológico vasco, ASJU. XIII, XIX, XXIV, XXVI, XXX, XXXVII, T. III, Saint-Sébastien, 1989-1993, pp. [40-41].

35. "Miscellanea toponymica et anthroponymica (III)", De re philologica linguae uasconicae $I V$, pp. 213-224, 1992.

36. Apellidos Vascos, $§ 119$

37. CARRÉ ALDAO, E., op cit.

38. GARCÍA ARIAS, X. LI., Pueblos asturianos : el porque de sus nombres, Éd.. Ayalga, Collección Popular Asturiana, Gijón, 1977, p. 98.

39. SAN MARTIN, J., "Introducción a la toponomástica guipuzcoana/Gipuzkoar toponomastikarako sarrera", in EUSKALTZAINDIA, Onomasticon Vasconiae 4. Actas de las I jornadas de onomástica, toponimia, Vitoria-Gasteiz, abril 1986// onomasika jardunaldien agiriak. Toponimia, Gasteiz, 1986ko apirila. Éd. Euskaltzaindia, 1991, p. 270.

40. RAVIER, X., "Le suffixe toponymique pyrénéen: “-un”.,., Via Domitia, 1963, pp. 64-68

41. "De la raiz *IZ- "agua" en vasco", FLV, nº 78, 1998, pp. 267-279.

42. MORALEJO LASSO, A., Toponimia gallega y leonesa, Éd. Pico sacro, Saint-Jacques-deCompostelle, 1977, p. 66.

43. Toponimia gallega y leonesa. Éd. Pico sacro, Saint-Jacques-de-Compostelle, 1977, pp. 30-31, n. 20. L'auteur y cite Hubschmid, J., Studien zur iberorom. Wortgesch. u. Ortsnamenk.

44. "Notas de toponimia comparada en el valle de Ega y la comarca de la Oliva", in EUSKALTZAINDIA, Onomasticon Vasconiae 4. Actas de las I jomadas de onomástica, toponimia, Viloria-Gasteiz. abril 1986/I onomastika jardunaldien agiriak. Toponimia, Gasteiz, 1986ko apirila, Éd. Euskaltzaindia, 1991, p. 142. 506.

45. URTASUN ANTZANO, E., "Fiteroko hainbat toki izcm. Hausnarketa", FLV, n 76, 1997, pp. $497-$

46. GARCIA ARIAS, X., op. cit., p. 54.

47. GARCIA ARIAS, X., op. cit.. p. 54.

48. CARRÉ ALDAO, E., op cit.

49. "Sobre los topónimos Oca y su entorno", Symbolae Ludovico Mitxelena sepiuagenario oblatae. Université du Pays Basque (UPV-EHU), Instituto de Ciencias de la Antigüedad, Vitoria, 1985, pp. 10071017.

50. Eslaba aldeko euskararen azterketa toponimiaren bidez. Onomasticon vasconiae II, Euskaitzaindia, Bilbao, 1994, p. 984.

51. Materiales para una historia de la lengua vasca en su relación con la latina, Estudios Vascos, XIX, Université de Salamanque, 1945, rééd. Éd.. Txertoa, Saint-Sébastien, 1990, p. 204. 
52. Op. cit., p. 204.

53. Sobre el mundo ibérico-pirenaico : datos, nuevas ideas sobre el lberismo. Estudios Vascos, XVIII, Éd. Txertoa, Saint-Sébastien, 1988, p. 84.

54. Etnografia Histórica de Navarra, 1972, p. 456.

55. Prüfung. Prüfung der Untersuchungen über die Urbewohner Hispaniens vermittelst der Vaskischen Sprache, 1921, Imp. F. Dümmler, Berlin, 192 pages. Traduction espagnole de T. Aranzadi : "Examen de las investigaciones sobre los aborigenes de España mediante la lengua vasca, traducción directa del alemán ( 2 edición) por Telesforo de Aranzadi", RIEV, 1935, p. 515

56. "Una forma no investigada en la toponimia del sur de Francia y de la España septentrional (el sufijo -és, -iés)", traduit du français par J. M. Enguita, Archivo de Filologia Aragonesa, XL, 1955.

57. "Las referencias sobre los Vascones hasta el año 800 después de J. C.", RIEB, XVIII, 1927, pp. $225-240$

58. "Sobre los iberos y su lengua", Homenaje ofrecido a Menéndez Pidal, III, 1925, pp. 225-240.

59. Sobre el pasado de la lengua vasca, Coll. Auñamendi, Saint-Sébastien, 1964, p. 127.

60. "Lengua comun y dialectos vascos", AS.JU, XV, 1981, in Palabras y Textos, pp. 39-40.

61. "Variété dialectale et unification littèraire", in Euskal HerriakJPays Basques, T. II, Les cahiers de I'IFOREP n ${ }^{\circ} 57$, Paris, 1989, p. 18.

62. "Los estudios geográfico-históricos sobre el pais vasco y la dialectología", in Sabre la religión antigua y el calendario del pueblo vasco, Estudios Vascos, T. I, Éd. Txertoa, Saint-Sébastien, 1984, p. 296.

63. LOUANDRE, Ch., Commentaires de Jules César. Guerre des Gaules. Traduction nouvelle avec le texte, des notes et un index, Éd. E. Fasquelle, Paris, 1931, p. 135, I. III, XXVI.

64. SCHULTEN, A., Los cántabros y astures y su gtuerra con Roma. Madrid, 1943.

65. Suhre la lengua vasca. Estudios Vascos, T. IX, Éd.. Txertoa, 2éd, Saint-Sébastien, 1988, p. 54. 